\section{Publication de descriptifs techniques extraits de l'Atlas des bois tropicaux}

L'Atlas des bois tropicaux publié en 2016 par les Éditions Quæ en version française (aux formats papier, epub et pdf) puis en 2017 en version anglaise (aux formats epub et pdf) présente des informations technologiques sur les bois destinées à tous les professionnels qui transforment et mettent en œuvre des bois tempérés ou tropicaux. Il réunit les principales caractéristiques technologiques de 283 essences tropicales et 17 essences de régions tempérées les plus employées en Europe, associées à leurs principales utilisations. Sa réalisation a bénéficié du soutien financier de l'Organisation internationale des bois tropicaux (OIBT), et d'Agropolis Fondation pour la version anglaise. Les descriptifs techniques d'une sélection d'essences tropicales seront régulièrement publiés dans la revue Bois et Forêts des Tropiques.

Cet ouvrage est le fruit d'un travail collectif de l'Équipe Bois tropicaux du Cirad qui, depuis des décennies, a étudié dans ses laboratoires les caractéristiques technologiques de plus de 1200 espèces tropicales en réalisant plusieurs centaines de milliers d'essais. La collecte récurrente d'informations sur le terrain est venue compléter ces données pour constituer une base de connaissances qui se décline aujourd'hui sous différents types d'applications.

Outre sa fonction de mémoire collective et de réservoir d'informations pour alimenter des produits documentaires de vulgarisation sur la qualité des bois tropicaux (fiches ou guides techniques, atlas, logiciels, etc.), cette base de connaissances constitue un outil d'étude des relations entre les propriétés des bois et les usages des produits forestiers. Aujourd'hui, les recherches et les études conduites par les chercheurs et les techniciens de l'Unité de recherche BioWooEB du Cirad continuent à alimenter cette base de connaissances sur le comportement technologique et les potentialités d'utilisation d'un nombre toujours plus important d'espèces forestières issues des régions tropicales des quatre continents.

L'Atlas des bois tropicaux décrit les caractéristiques de 300 essences. C'est un outil de référence pour les opérateurs de la filière bois en France et à l'étranger, ainsi que pour les établissements de recherche et d'enseignement, les institutionnels, les donneurs d'ordre, les architectes, les maîtres d'œuvre, les maîtres d'ouvrage et, de façon générale, pour tous les professionnels qui transforment et mettent en œuvre des bois tempérés ou tropicaux, ou qui projettent de le faire. Pour chaque essence, le descriptif technique reprend les informations suivantes : famille et appellation(s) botanique(s), origine géographique, éventuelle restriction commerciale selon la convention de Washington (CITES), description de la grume, description du bois, principales propriétés physiques et mécaniques, durabilité naturelle et imprégnabilité du bois, traitement de préservation, séchage, sciage et affûtage, assemblage, classements commerciaux, réaction au feu, principales utilisations, principales appellations vernaculaires. Chaque descriptif est illustré par deux photos de débit sur dosse et sur quartier (ou faux quartier), par deux macrophotographies aux grossissements 20 et 115 et par une photo d'un ouvrage construit avec le bois décrit.

Les détails sur le contenu de ces descriptifs techniques, sur la nature et l'intérêt des informations, des caractéristiques et des données qu'ils contiennent sont disponibles dans une notice d'informations générales sur le site web de Bois et Forêts des Tropiques, annexée uniquement à la version web de cet article.

Jean GÉRARD

Rédacteur scientifique de la revue Bois et Forêts des Tropiques et chercheur de l'Unité de recherche BioWooEB

Référence complète et accès à l'ouvrage :

J. Gérard (coord.), D. Guibal (au.), J.-C. Cerre (au.), S. Paradis (au.), et 40 auteurs, 2016. Atlas des bois tropicaux - Caractéristiques technologiques et utilisations. Éditions Quæ, 1000 p. https://www.quae. com/produit/1408/9782759225521/atlas-des-bois-tropicaux

\section{Doi et accès à la notice d'information générale :}

https://doi.org/10.19182/bft2021.347.a36353

Droit d'auteur (c) 2021, Bois et Forêts des Tropiques (c) Cirad

Date de publication : 25 février 2021

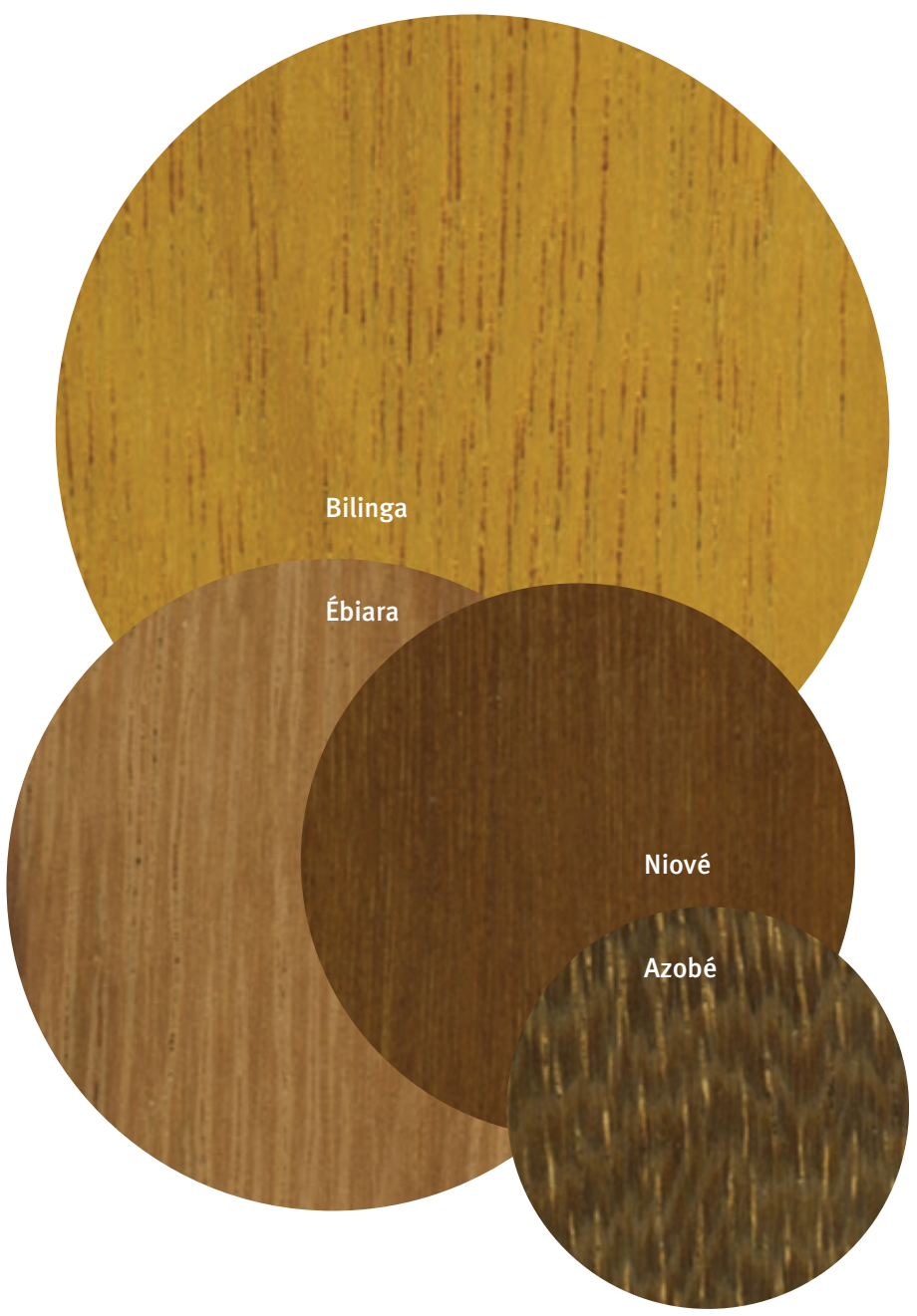




\section{Publication of technical descriptions from the Tropical Timber Atlas}

The Tropical Timber Atlas, published in 2016 by Éditions Quæ in French (in print, epub and pdf formats) and in 2017 in English (in epub and pdf formats), presents technological information on wood for all professionals who process and use wood from temperate or tropical species. It covers the main technological characteristics of 283 tropical species and 17 temperate species most commonly used in Europe, together with their main uses. It was produced with financial support from the International Tropical Timber Organization (ITTO) and from the Agropolis Foundation for the English version. Technical descriptions of a selection of tropical species will be published regularly in the Bois et Forêts des Tropiques journal.

This book is the outcome of a collective effort by CIRAD's Tropical Timber Team, which for decades has been studying the technological characteristics of more than 1,200 tropical species in its laboratories and has conducted several hundred thousand trials. Recurrent information gathering in the field has supplemented this data to build up a knowledge base that is now applied to a variety of uses.

As well as serving as a collective memory and a repository of information for the production of documentation for non-specialists on the quality of tropical timber (technical guides and data sheets, atlases, software, etc.), this knowledge base is a tool for studying the relationships between wood properties and the uses of forest products.

The studies conducted by researchers and technicians working for CIRAD's BioWooEB Research Unit are continuing to contribute to this knowledge base on the technological behaviour and potential uses of an ever-increasing number of forest species from tropical regions on four continents.

The Tropical Timber Atlas describes the characteristics of 300 species. It is a reference tool for operators in the timber industry across the world, as well as for researchers and teachers, institutions, contractors, architects, project managers and, in general, for all professionals who process and use temperate or tropical woods or plan to do so.

For each species, the technical description includes the following: botanical family and name(s), geographical origin, possible trade restrictions under the Washington Convention (CITES), description of the timber log, description of the wood, its main physical and mechanical properties, natural durability and impregnability, wood preservation treatment, drying, sawing and sharpening, assembly, commercial classifications, reaction to fire, main uses, main vernacular names. Each description is illustrated with photos of the wood sawn in the length and half-quarter sawn (or false quarter), two macro-photographs at 20 and 115 magnifications and a photo of a structure built with the wood described.
Details of the content of these technical descriptions, the type of information and areas of relevance and the characteristics and data they contain are available in a general information leaflet on the Bois et Forêts des Tropiques website, which is attached to the web version only of this article.

Jean GÉRARD

Scientific editor of the Bois et Forêts des Tropiques journal and researcher at the BioWooEB Research Unit

Full reference and access to the book:

J. Gérard (coord.), D. Guibal (au.), J.-C. Cerre (au.), S. Paradis (au.), and 40 authors, 2016. Tropical Timber Atlas - Technological characteristics and uses. Publisher: Éditions Quæ, 1000 p. https://www. quae.com/produit/1477/9782759227716/tropical-timber-atlas

Doi and access to the general information leaflet: https://doi.org/10.19182/bft2021.347.a36353

Droit d'auteur (c) 2021, Bois et Forêts des Tropiques (c) Cirad Date de publication : 25 février 2021

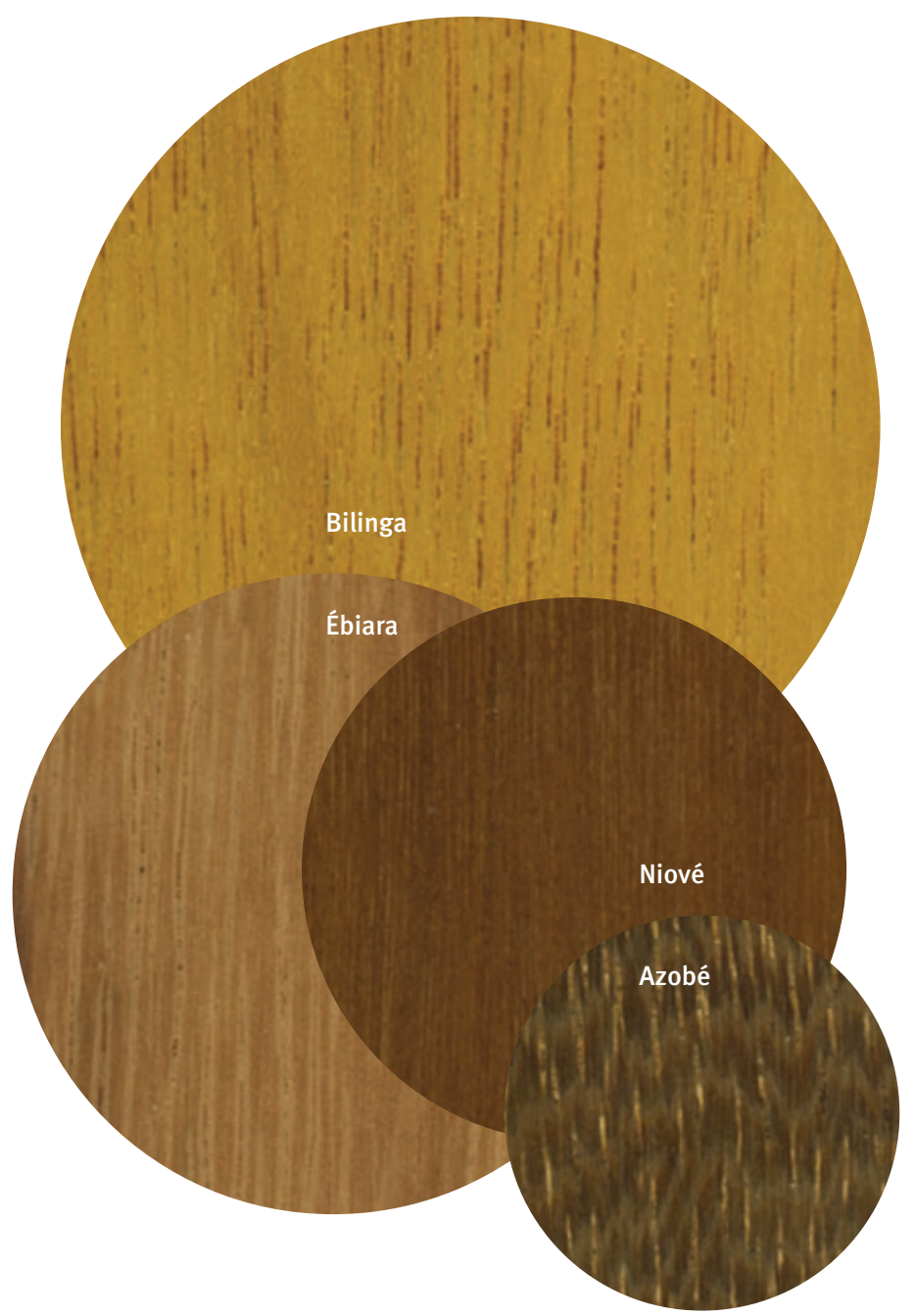




\section{Addendum extrait de l'Atlas des bois tropicaux : Guide d'utilisation : informations générales}

\section{Dénominations et CITES}

\section{Nom pilote des bois}

Les noms pilotes retenus proviennent de la Nomenclature générale des bois tropicaux publiée par l'Association technique internationale des bois tropicaux (ATIBT) en 2016. Cet ouvrage de référence est reconnu internationalement, notamment en Europe pour la mise en œuvre du Règlement sur le bois de I'Union européenne (RBUE).

Pour quelques essences, le nom pilote de la nomenclature, qui est I'appellation de référence, est couplé à une seconde appellation commerciale fréquemment utilisée, identifiée par un astérisque $\left({ }^{*}\right)$. Les essences concernées par cette double appellation sont les suivantes: Alan / Alan-Batu*; Anzèm / Nténé*; Balau Yellow / Bangkirai*; Catucaém / Louro faia*; Coraçao de negro*/Panacoco; Cryptomeria*/Sugi ; Dukali / Amapa* ; Fuma / Fromager* ; Grenadillo / Grenadille d'Afrique* ; Kurokaï / Breu* ; Mango/Machang*; Manniballi / Manil montagne*; Pashaco/Paricá*; Pinus kesiya*/Kesiya Pine; Pinus merkusii*/Merkusii Pine; Pinus patula*/Patula Pine; Teck* / Teak.

\section{Famille et noms botaniques}

Comme les noms pilotes, la famille et les noms botaniques mentionnés sont également réfé-rencés dans la Nomenclature générale des bois tropicaux (ATIBT, 2016).

Certains noms botaniques sont associés aux abréviations suivantes :

- «spp. » (species pluralis) et " p.p. » (pro parte). Selon les codes de botanique, l'abréviation "spp. » signifie plus d'une espèce du genre et peut inclure toutes les espèces du genre ce qui peut prêter à confusion. En effet, suivant les différents auteurs, cette abreviation est utilisée soit pour désigner de façon non exhaustive plusieurs espèces d'un même genre, soit pour désigner l'ensemble des espèces du genre.

De ce fait, dans cet Atlas, I'abréviation «p.p. » est utilisée pour les essences de bois regroupant plusieurs espèces du même genre sans que la totalité des espèces de ce genre ne soit concernée.

- «subgen. » (sous-genre). Dans un genre donné, plusieurs espèces ayant une grande proximité botanique peuvent être regroupées dans un sous-genre. Dans un sous-genre, l'essence peut correspondre à toutes les espèces, à plusieurs espèces non listées exhaustivement ou à une partie d'entre elles, selon les mêmes règles que celles précédemment énoncées.

\section{CITES (Convention de Washington, 2016)}

La CITES (Convention on International Trade in Endangered Species of Wild Fauna and Flora ou « Convention de Washington») est un accord international entre États. Cet accord a pour but de veiller à ce que le commerce international d'animaux et de plantes sauvages ne menace pas la survie de leurs espèces. Les bois tropicaux sont donc concernés.

Les espèces soumises à la réglementation sont inscrites dans l'une des trois annexes de la convention :

- I'annexe I comprend toutes les espèces menacées d'extinction. Le commerce de leurs spécimens n'est autorisé que dans des conditions exceptionnelles;

- I'annexe II comprend toutes les espèces qui ne sont pas nécessairement menacées d'extinction, mais dont le commerce des spécimens doit être réglementé pour éviter une exploitation incompatible avec leur survie ; 
- I'annexe III comprend toutes les espèces protégées dans un pays. Ce pays doit demander I'assistance des autres Parties à la CITES pour en contrôler le commerce. La procédure à suivre pour procéder à des changements dans I'annexe III est distincte de celle des annexes I et II car chaque Partie est habilitée à y apporter unilatéralement des amendements.

La mention « pas de restriction commerciale » correspond aux espèces non inscrites à la CITES. D'autres informations sont disponibles sur le site de la CITES : www.cites.org/fra.

\section{Description de la grume}

\section{Diamètre}

La gamme de valeurs mentionnée correspond aux diamètres des grumes les plus souvent exploitées. Ces valeurs sont à comparer aux DME (diamètre minimum d'exploitabilité) ou DMA (diamètre minimum d'aménagement) déterminés par chaque pays producteur pour assurer une reconstitution suffisante de la forêt sur la durée de la rotation. Les valeurs de DME et DMA sont généralement disponibles auprès des services forestiers de ces pays.

\section{Épaisseur de l'aubier}

La fourchette de valeurs mentionnée correspond aux épaisseurs d'aubier les plus fréquemment rencontrées.

\section{Flottabilité}

Pour les essences tropicales, deux classes " (bois flottable», " bois non flottable ») ont été définies en fonction de la densité moyenne des bois à l'état vert (" après abattage »). Une troisième classe (« sans objet ») a été définie pour les essences tempérées.

\section{Conservation en forêt}

Selon la durabilité naturelle des bois, la conservation en forêt est estimée faible (traitement nécessaire), moyenne (traitement recommandé) ou bonne. La notion de conservation en forêt ne concerne que le duramen car l'aubier est toujours considéré comme non durable.

\section{Description du bois}

\section{Couleur}

Bien que la couleur et l'aspect d'un bois soient en général spécifiques à une essence donnée, la couleur n'est pas un facteur constant d'un arbre à un autre ou d'une pièce de bois à une autre pour la même espèce. Elle peut varier en fonction de différents paramètres et évolue dans le temps. Les variations de pente de fil (par exemple, contrefil, fil ondulé) et du taux d'humidité du bois peuvent altérer la perception de la couleur.

Chaque essence est caractérisée par une couleur de référence choisie parmi 18 couleurs prédéfinies : blanc, blanc crème, blanc rosé, jaune clair, jaune, jaune orangé, brun clair, brun, brun jaune, brun rosé, brun rouge, brun foncé, rouge clair, rouge, rouge foncé, gris, noir et violet. Le descriptif de la couleur des bois mentionné dans la note de la rubrique « Description du bois " met l'accent sur la plage de variation rencontrée. II ne prend pas en compte, entre autres, l'évolution de la couleur des bois exposés aux intempéries.

\section{Aubier}

L'aubier peut être bien distinct, peu distinct ou non distinct. Dans le cas où il n'y pas d'information disponible, la notation «n.d.» a été adoptée.

\section{Grain}

Le grain d'un bois correspond à l'impression visuelle donnée par la taille et la disposition des vaisseaux. Trois classes de grain sont définies : fin, moyen et grossier.

\section{Fil et contrefil}

Le fil du bois correspond à l'orientation générale des fibres par rapport à l'axe de la grume. Le contrefil est dû à une inclinaison alternée (par rapport à l'axe du tronc) des couches successives de bois qui se forment durant la croissance de l'arbre. 


\section{Propriétés physiques et mécaniques}

Les valeurs des propriétés physiques et mécaniques (valeurs moyennes) sont issues des essais réalisés dans les laboratoires du Cirad ou de la littérature internationale. Elles sont à utiliser avec prudence, compte tenu du caractère très variable des propriétés des bois. Cette variabilité est bien connue de tous les professionnels. Elle dépend de nombreux facteurs externes ou internes : âge des arbres, position du bois à l'intérieur du tronc, maturité du bois et conditions de croissance (type de sol, pluviométrie, climat...).

\section{Densité}

La densité ou densité relative d'un solide est le rapport entre sa masse volumique et la masse volumique de l'eau (eau pure à $4{ }^{\circ} \mathrm{C}$ et à pression atmosphérique normale, soit $1000 \mathrm{~kg} / \mathrm{m}^{3}$ ). Elle n'a pas d'unité.

La densité indiquée est déterminée sur les bois à $12 \%$ d'humidité. C'est une caractéristique technologique de base qui est la première à être déterminée pour qualifier un bois. Cette propriété est reliée plus ou moins étroitement avec les principales propriétés physiques et mécaniques du bois, ainsi qu'avec certaines caractéristiques de mise en œuvre.

\section{Dureté Monnin}

La dureté Monnin (déterminée sur les bois à 12 \% d'humidité) est une propriété particulièrement importante à connaître lorsque les bois sont utilisés en revêtement de sol (parquet, plancher et platelage), mais aussi pour tout autre emploi dans lequel le bois est soumis à des chocs ou à des poinçonnements. Elle n'a pas d'unité.

Classes de dureté :

- $\mathrm{D} \leq 1,5$ : bois très tendre ;

- $1,5 \leq \mathrm{D} \leq 3$ : bois tendre ;

- $3 \leq \mathrm{D} \leq 6$ : bois mi-dur ;

- $6 \leq \mathrm{D} \leq 9$ : bois dur ;

- $\mathrm{D} \geq 9$ : bois très dur.

La méthode de détermination de la dureté Monnin est définie dans la norme française NF B 51-013 (1985).

La dureté Janka est une caractéristique utilisée par plusieurs pays. Elle est associée à une autre méthode de détermination. Sallenave (1971) propose la relation suivante entre la dureté Monnin et la dureté Janka :

dureté Janka (en livre anglaise) $=300 \times$ dureté Monnin.

\section{Point de saturation des fibres (PSF, en \%)}

Dans un bois vert, une partie de l'eau remplit plus ou moins complètement les vides cellulaires et intercellulaires. L'évacuation de cette eau libre s'effectue sans retrait du bois. Lorsque l'eau libre a entièrement disparu, le bois ne contient plus que de l'eau liée chimiquement aux parois des cellules. Son élimination lors du séchage occasionne des phénomènes de retrait à l'origine de déformations du bois.

Le point de saturation des fibres correspond au taux d'humidité du bois saturé en eau liée. En dessous de ce taux, le bois commence à se contracter en séchant. Le PSF varie le plus souvent entre $20 \%$ et $40 \%$ suivant les essences, mais se situe généralement aux environs de $30 \%$.

Classes de point de saturation des fibres :

- PSF $\leq 25 \%$ : faible ;

- $25 \% \leq$ PSF $\leq 35 \%$ : moyen ;

- PSF $\geq 35 \%$ : élevé.

\section{Coefficient de retrait volumique (Rv, en \% par \%)}

Lorsqu'une pièce de bois sèche en dessous de son point de saturation des fibres (PSF), son volume diminue. Si elle reprend de l'humidité, son volume augmente jusqu'au PSF. Si la reprise d'humidité se poursuit au-dessus du PSF, le volume ne varie plus. Le coefficient de 
retrait volumique (Rv) est utilisé pour quantifier ces variations de volume, il correspond au retrait volumique d'une pièce de bois lorsque son humidité varie de $1 \%$.

Classes de coefficient de retrait volumique :

- $\mathrm{Rv} \leq 0,35$ : retrait faible ;

- $0,35 \leq \operatorname{Rv} \leq 0,55$ : retrait moyen ;

- $R v \geq 0,55$ : retrait élevé.

Retrait tangentiel total (Rt) et retrait radial total (Rr) (en \%)

Jusqu'au point de saturation des fibres (PSF), le bois ne se rétracte pas en séchant. En dessous de ce seuil, il est soumis à des variations dimensionnelles quand son humidité varie. Le retrait en dessous du PSF intervient suivant les trois directions du bois : longitudinale, tangentielle et radiale.

Le retrait longitudinal est très faible par rapport au retrait tangentiel et au retrait radial, de l'ordre de quelques dixièmes de pour cent. Cependant, il peut avoir une influence notable sur les variations dimensionnelles de pièces de grande longueur. Très peu de données sont disponibles sur cette caractéristique qui reste délicate à mesurer en laboratoire.

Le retrait tangentiel total (Rt) et le retrait radial total $(\mathrm{Rr})$ sont habituellement déterminés pour qualifier le comportement du bois lors du séchage ou plus généralement lors des variations d'humidité.

Classes de retrait tangentiel total (Rt) :

- Rt $\leq 6,5 \%$ : retrait faible ;

- $6,5 \% \leq \mathrm{Rt} \leq 10 \%$ : retrait moyen ;

- Rt $\geq 10 \%$ : retrait élevé.

Classes de retrait radial total $(\mathrm{Rr})$ :

- $\operatorname{Rr} \leq 3,8 \%$ : retrait faible ;

- $3,8 \% \leq \operatorname{Rr} \leq 6,5 \%$ : retrait moyen ;

- $\operatorname{Rr} \geq 6,5 \%$ : retrait élevé.

\section{Quotient de retrait $\mathrm{Rt} / \mathrm{Rr}$}

Le quotient entre le retrait tangentiel total et le retrait radial total ( $\mathrm{Rt} / \mathrm{Rr})$ donne une indication sur les déformations subies par une pièce de bois soumise à des variations d'humidité.

Ce paramètre est particulièrement important pour les débits non orientés (débits sur faux quartier). Un rapport Rt / Rr qui tend vers une valeur supérieure ou égale à 2 indique qu'une essence est sensible aux déformations. Plus cette valeur est proche de 1, plus le bois est stable quel que soit le type de débit.

\section{Conductivité thermique}

La conductivité thermique $\lambda$ (Watt par mètre et par Kelvin, W/m.K) d'un matériau caractérise son aptitude à conduire la chaleur. $\lambda$ est d'autant plus faible que le matériau est isolant.

Les valeurs de $\lambda$ mentionnées dans cet ouvrage pour chaque essence sont issues d'une campagne de mesures sur un vaste échantillonnage d'essences tropicales et tempérées et une large gamme de densités. Cependant, cet échantillonnage ne couvre pas toutes les essences décrites.

Les mesures ont été réalisées au laboratoire de thermo-physique du Groupe d'études des matériaux hétérogènes (GEMH, Centre européen de la céramique, Limoges) en utilisant la méthode du disque chaud (hot disk) régie par la norme NF EN ISO 22007-2 (octobre 2015). Cette campagne de mesure a révélé que la conductivité thermique $(\lambda)$ est corrélée à la densité (D) des bois (figure 1).

La conductivité thermique $\lambda$ est donc reliée à la densité $D$ par l'équation : $\lambda=0,289 D+0,030$.

Les valeurs de $\lambda$ mentionnées dans cet ouvrage ont été déterminées à l'aide de ce modèle, en utilisant la densité moyenne de chaque essence. 


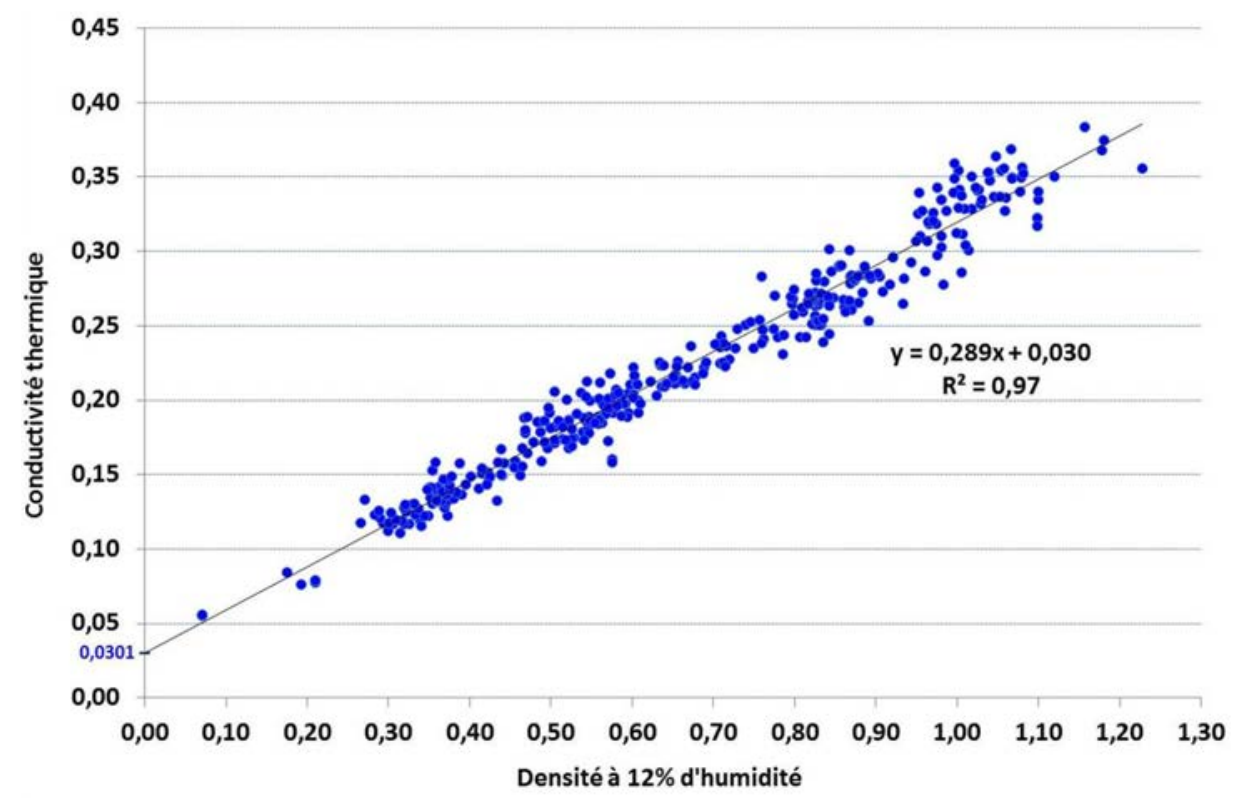

Figure 1. Relation entre la conductivité thermique et la densité du bois (mesurée à $12 \%$ d'humidité).

\section{Pouvoir calorifique}

Le pouvoir calorifique d'un matériau correspond à la quantité de chaleur dégagée par sa combustion. Il s'exprime le plus souvent en kilojoule par kilogramme (kJ/kg) ou en joule par gramme (J/g), éventuellement en calories par gramme (cal/g) ou en kilocalories par kilogramme ( $\mathrm{kcal} / \mathrm{kg})$. Il est possible de déterminer deux types de pouvoir calorifique :

- le pouvoir calorifique supérieur (PCS) est la quantité de chaleur dégagée par la combustion, à volume constant, de $1 \mathrm{~kg}$ de matière anhydre. L'eau formée au cours de la combustion est condensée, alors que la chaleur libérée par la condensation de l'eau (chaleur latente de l'eau) est récupérée ;

- le pouvoir calorifique inférieur (PCI) est la quantité d'énergie dégagée par la combustion à pression constante (à l'air libre) de $1 \mathrm{~kg}$ de matière anhydre. La vapeur d'eau formée au cours de la combustion n'est pas condensée. L'énergie de condensation n'est donc pas récupérée.

Le PCl est la grandeur utilisée couramment pour les calculs de combustion. Il est exprimé en $\mathrm{kJ} / \mathrm{kg}$ de bois anhydre.

Le PCS est déterminé expérimentalement au laboratoire à l'aide d'un appareil appelé « bombe calorimétrique ».

Pour le bois, le pouvoir calorifique inférieur $(\mathrm{PCl}$, en $\mathrm{kJ} / \mathrm{kg}$ ) est déduit du pouvoir calorifique supérieur (PCS) par la formule suivante :

$\mathrm{PCl}$ anhydre $=$ PCS anhydre $-(212,2 \times \mathrm{H})$

où $\mathrm{H}$ est la teneur en hydrogène (exprimée en pourcentage massique) de la biomasse considérée (loi de passage déterminée à partir de la norme NF EN 14918 mars 2010 intitulée "Biocombustibles solides, détermination du pouvoir calorifique »).

Les $\mathrm{PCI}$ mentionnés dans cet ouvrage ont été déterminés à partir des valeurs de PCS mesurées en laboratoire au Cirad. La teneur en hydrogène $\mathrm{H}$ n'étant pas déterminée lors de l'essai, une valeur moyenne de $\mathrm{H}$ égale à $5,85 \%$ a été retenue pour le calcul (l'expérience montre que la valeur de $\mathrm{H}$ est quasi constante d'une essence à une autre). 


\section{Contrainte de rupture en compression (en MPa)}

Cette contrainte (notée $\mathrm{C}_{12}$ ) est déterminée sur les bois à $12 \%$ d'humidité selon la procédure définie dans la norme NF B 51-007 (septembre 1985). Elle résulte de l'effort qu'il faut appli-quer suivant la direction du fil du bois pour obtenir la rupture d'une éprouvette de dimensions standard.

Classes de contrainte en compression parallèle :

- $\mathrm{C} 12 \leq 45 \mathrm{MPa}$ : contrainte faible ;

- $45 \mathrm{MPa} \leq \mathrm{C} 12 \leq 75 \mathrm{MPa}$ : contrainte moyenne ;

- C12 $\geq 75$ MPa : contrainte élevée.

\section{Contrainte de rupture en flexion statique (en MPa)}

La contrainte en flexion statique (notée $\mathrm{F}_{12}$ ) est déterminée sur les bois à $12 \%$ d'humidité selon la procédure définie dans la norme B 51-008 (novembre 1987). Elle résulte de l'effort qu'il faut appliquer dans la zone centrale d'une éprouvette de dimension standard reposant sur deux appuis pour atteindre sa rupture.

Classes de contrainte en flexion statique :

- $\mathrm{F}_{12} \leq 75 \mathrm{MPa}$ : contrainte faible ;

- $75 \mathrm{MPa} \leq \mathrm{F}_{12} \leq 125 \mathrm{MPa}$ : contrainte moyenne ;

- $\mathrm{F}_{12} \geq 125 \mathrm{MPa}$ : contrainte élevée.

\section{Module d'élasticité longitudinal (en MPa)}

Le module d'élasticité longitudinal $\left(E_{\mathrm{L}}\right)$ est déterminé sur les bois à $12 \%$ d'humidité ; c'est une propriété de première nécessité technologique pour les emplois en structure dans lesquels les pièces de bois sont fréquemment sollicitées en flexion statique. Cette propriété caractérise la proportionnalité entre la charge et la déformation. Elle constitue un indicateur de la rigidité du bois.

Classes de module d'élasticité longitudinal :

- $\mathrm{E}_{\mathrm{L}} \leq 12500 \mathrm{MPa}$ : module faible ;

- $12500 \mathrm{MPa} \leq \mathrm{E} \leq 18500 \mathrm{MPa}$ : module moyen ;

- $\mathrm{E}_{\mathrm{L}} \geq 18500 \mathrm{MPa}$ : module élevé.

\section{Durabilité naturelle et imprégnabilité du bois}

Sauf mention particulière relative à l'aubier, les caractéristiques de durabilité concernent le duramen des bois arrivés à maturité. L'aubier doit toujours être considéré comme non durable vis-à-vis des agents de dégradation biologique du bois. Un bois dont l'humidité en service est inférieure à environ $20 \%$ présente peu de risques d'être attaqué par les champignons. Des températures inférieures à $5{ }^{\circ} \mathrm{C}$ environ empêchent tout développement des champignons. De même, des bois immergés ou portés à des températures élevées (de l'ordre de $60^{\circ} \mathrm{C}$ ) ne sont jamais attaqués par les champignons quelle que soit leur durabilité naturelle.

\section{Résistance aux champignons}

La résistance des bois aux champignons est déterminée sur des échantillons de dimensions normalisées mis en présence de souches de champignons dans des conditions ambiantes contrôlées. Ces essais durent plusieurs mois.

La norme NF EN 350, en cours de révision au moment de l'édition de cet ouvrage, définit des classes de durabilité naturelle du bois contre des champignons lignivores :

- bois très durables : classe DC1 (durability class 1), nommée " classe 1 »;

- bois durables : classe DC2, nommée « classe 2 »;

- bois moyennement durables : classe DC3, nommée « classe 3 »;

- bois faiblement durables : classe DC4, nommée « classe 4 »;

- bois non durables : classe DC5, nommée « classe 5 ». 


\section{Résistance aux insectes des bois secs (lyctus, bostryches, vrillettes)}

La grande majorité des bois tropicaux commercialisés n'est pas attaquée par les insectes de bois sec, à condition que ces bois soient mis en œuvre sans aubier. Lorsque l'aubier est peu distinct, il est préférable de traiter les bois contre les insectes de bois sec. Certaines essences tropicales sont attaquées dans la totalité du bois et demandent des précautions particulières à l'état sec. Les bois sciés ou les produits finis ne sont attaqués que s'ils contiennent encore de l'aubier et une teneur en amidon suffisante.

Selon la norme NF EN 350, une essence est classée sensible (classe DC S, nommée « classe S ») si elle est attaquée pendant l'essai mené en laboratoire. Dans le cas contraire, elle est considérée comme durable (classe DC D, nommée « classe D »).

\section{Résistance aux termites}

Les conditions de détermination de la résistance des bois aux termites sont analogues à celles de la résistance aux champignons. Des échantillons de dimension normalisée sont mis en présence de termites. L'intensité de l'attaque des termites et, par conséquent, la résistance naturelle des bois sont quantifiées en mesurant la profondeur de pénétration des termites dans l'échantillon. La norme NF EN 350 définit trois classes de durabilité naturelle vis-à-vis des termites :

- bois durables : classe DC D (durability class D), nommée "classe D »;

- bois moyennement durables : classe DC M, nommée " classe $M$ »;

- bois sensibles : classe DC $S$, nommée « classe $S$ ».

\section{Imprégnabilité du duramen}

L'imprégnabilité d'un bois correspond à son aptitude à être imprégné par un produit de préservation. La norme NF EN 350 définit quatre classes d'imprégnabilité :

- classe 1, imprégnable. Le bois scié peut être imprégné complètement et sans difficulté par un traitement sous pression ;

- classe 2, moyennement imprégnable. Habituellement, une pénétration complète du produit n'est pas possible. Cependant, après un traitement sous pression durant deux ou trois heures, une pénétration latérale de plus de $6 \mathrm{~mm}$ peut être obtenue chez les résineux. Chez les feuillus, une large proportion de la structure (vaisseaux et rayons ligneux) peut être imprégnée ;

- classe 3, peu imprégnable. Un traitement sous pression durant trois ou quatre heures ne permet pas une pénétration latérale du produit de plus de 3 à $6 \mathrm{~mm}$;

- classe 4, non imprégnable. Peu de produit de préservation absorbé, même après 3 ou 4 heures de traitement sous pression. La pénétration latérale et longitudinale est très faible.

\section{Classe d'emploi}

La classe d'emploi correspond à un degré d'exposition aux différents agents de dégradation biologique découlant d'une situation en service d'un élément ou d'un ouvrage en bois. Elle peut changer après modification de la conception ou de la situation de l'ouvrage. Elle ne définit pas systématiquement une durée de service, mais seulement les conditions d'une attaque biologique potentielle. Dans une classe d'emploi, les spécifications de traitement et le choix de l'essence ont une incidence directe sur la durée de service.

La durée de service doit donc être interprétée en fonction des essences et de la sévérité des expositions. Elle dépend de la durabilité naturelle du bois, mais aussi d'autres facteurs tels que les détails de la conception d'un ouvrage (risques de pièges à eau, ventilation du bois...), la nature des entretiens prévus et les conditions climatiques locales.

L'utilisation d'un bois, dont la durabilité naturelle est supérieure à celle qui est préconisée par la norme NF EN 460 (juillet 1994) pour un emploi donné, permet d'allonger la durée de service de l'ouvrage. Réciproquement, pour des éléments d'ouvrage à durée de vie très courte (construction provisoire), des essences de durabilité naturelle inférieure à celle mentionnée dans la norme EN 460 peuvent être préconisées. 
Note. Ne pas confondre les notions de « classe de résistance aux champignons » et de « classe d'emploi » dont les barèmes de qualification sont différents.

Les situations en service ont été regroupées en classes d'emplois (norme NF EN 335, mai 2013). Chaque classe correspond à une catégorie d'utilisations associée à des risques de dégradation biologique de même niveau.

\section{Catégories regroupant les classes selon les conditions d'emploi}

\begin{tabular}{|c|c|}
\hline $\begin{array}{l}\text { Classe } \\
\text { d'emploi }\end{array}$ & Usage général \\
\hline 1 & À l'intérieur, au sec \\
\hline 2 & À l'intérieur ou sous abri, pas d'exposition aux intempéries. Possibilité de condensation d'eau \\
\hline 3 & $\begin{array}{l}\text { À l'extérieur, au-dessus du sol, exposé aux intempéries. La classe } 3 \text { peut être est subdivisée en } 2 \\
\text { sous-classes : } 3.1 \text { Conditions d'humidification courtes - } 3.2 \text { Conditions d'humidification prolongées }\end{array}$ \\
\hline 4 & À l'extérieur en contact avec le sol ou l'eau douce \\
\hline 5 & Immergé dans l'eau salée de manière régulière ou permanente \\
\hline
\end{tabular}

Spécificités de la classe 5 . L'appartenance d'une essence à la classe 5 est mentionnée séparément. Une essence qui couvre la classe 5 couvre généralement la classe 4, excepté quelques rares essences ne couvrant que la classe 3 ou la classe 2 (Basralocus, Garapa, Iroko, Louro vermelho, Sougué).

La norme européenne NF EN 460 (juillet 1994) propose un tableau de correspondance entre le niveau de durabilité naturelle des bois massifs et leurs possibilités d'emploi dans une classe de risque donnée (tableau ci-dessous). Cette norme est antérieure au remplacement de la notion de " classe de risque » par celle de « classe d'emploi » (NF EN 335, mai 2013), ces deux notions étant quasiment équivalentes.

\section{Classes de durabilité naturelle selon la classe de risque}

\begin{tabular}{|c|c|c|c|c|c|}
\hline \multirow{2}{*}{$\begin{array}{l}\text { Classe de risque couverte } \\
\text { par la durabilité naturelle }\end{array}$} & \multicolumn{5}{|c|}{ Classe de durabilité naturelle } \\
\hline & 1 & 2 & 3 & 4 & 5 \\
\hline 1 & Oui $i^{(1)}$ & Oui & Oui & Oui & Oui \\
\hline 2 & Oui & Oui & Oui & Oui mais & Oui mais \\
\hline 3 & Oui & Oui & Oui mais & Au cas par cas ${ }^{(3)}$ & Au cas par cas \\
\hline 4 & Oui & Oui mais ${ }^{(2)}$ & Non mais ${ }^{(4)}$ & $\operatorname{Non}^{(5)}$ & Non \\
\hline 5 & Oui & Non mais & Non mais & Non & Non \\
\hline
\end{tabular}

(1) Oui : la durabilité naturelle couvre la classe de risque.

(2) Oui mais : la durabilité naturelle couvre normalement la classe de risque. Mais pour certains emplois, un traitement de préservation peut être recommandé.

(3) Au cas par cas : la durabilité naturelle peut être suffisante. Mais en fonction de l'essence du bois, de sa perméabilité et de son emploi final, un traitement de préservation peut être nécessaire.

(4) Non mais : un traitement de préservation est normalement recommandé. Mais pour certains emplois, la durabilité naturelle peut être suffisante pour couvrir la classe de risque.

(5) Non : la durabilité naturelle ne couvre pas la classe de risque ; un traitement de préservation est nécessaire.

Pour les classes de risque 2 à 5 , les correspondances ne sont pas définies de manière précise pour certains niveaux de durabilité. Pour certaines essences, les classes d'emploi ne sont données qu'à titre indicatif. Les valeurs correspondantes doivent être utilisées avec précaution et professionnalisme.

\section{Traitement de préservation}

Cette section préconise des traitements ou des précautions d'utilisation d'une essence en cas de risques d'attaques d'insectes de bois sec, ainsi qu'en cas d'humidification temporaire ou permanente. 


\section{Séchage}

Le comportement général du bois durant son séchage artificiel est décrit et évalué qualitativement. Pour certaines essences, les précautions d'usage sont rappelées pour assurer une bonne qualité de séchage.

\section{Risque de déformation}

Les déformations des bois débités peuvent être de 4 types (figure 2).
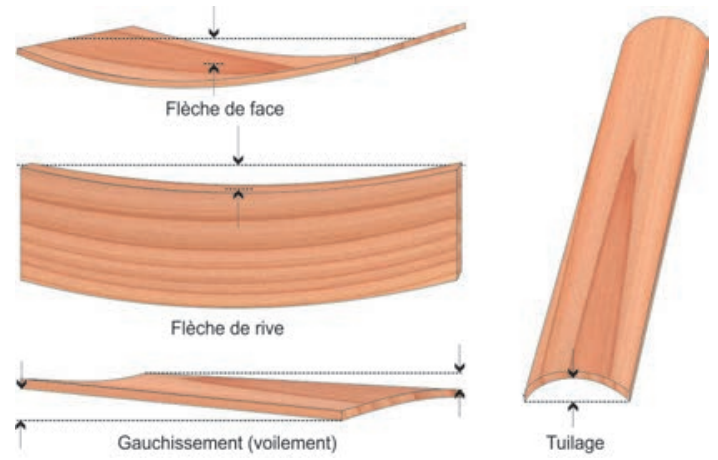

Figure 2. Déformations des bois.

\section{Risque de cémentation}

Le phénomène de cémentation désigne le blocage du transfert de l'eau de l'intérieur vers l'extérieur d'une pièce de bois. Il est dû à une modification de la structure des cellules du bois en périphérie. Il est souvent causé par un séchage trop brutal provoquant un dessèchement trop intense en surface. La cémentation peut s'accompagner de la formation de poches d'eau au cœur des pièces de bois.

\section{Risque de fentes}

Les fentes peuvent apparaître à la surface ou aux extrémités des pièces de bois. Elles peuvent aussi se former à l'intérieur des bois sciés (fentes internes).

\section{Risque de collapse}

Le collapse correspond à un effondrement associé à une déformation des cellules du bois durant les premières phases du séchage. Ce défaut se développe avant que le bois n'ait atteint le point de saturation des fibres (PSF). Il se manifeste sous forme d'ondulations des faces de sciage ou de déformations complètes de l'état de surface des pièces de bois, avec ou sans fentes internes.

\section{Programmes de séchage}

Un programme de séchage définit les enchaînements de conditions climatiques à respecter dans la cellule du séchoir. Durant tout le cycle de séchage du bois, le passage d'une ambiance climatique donnée à la suivante est conditionné par la baisse du taux d'humidité du bois.

Pour chaque essence, un des neuf programmes de séchage présentés à la fin de cette section est proposé à titre indicatif pour une mise en application dans un séchoir à air chaud climatisé (ACC).

Chaque programme est constitué de cinq phases successives : deux phases de préchauffage, le séchage, l'équilibrage et le refroidissement.

Les programmes sont définis par cinq paramètres :

1. la durée de chaque phase (en heure);

2. le taux d'humidité du bois $\mathrm{H} \%$ mesuré le plus souvent à l'aide de sondes enfoncées dans plusieurs pièces de bois réparties dans la cellule, selon une méthode électrique ayant le 
même principe que l'humidimètre à pointes. Le pilotage du séchage peut se faire sur la moyenne des valeurs enregistrées par les sondes, sur la valeur enregistrée la plus élevée ou sur la valeur la plus basse. Les sondes indiquant des valeurs aberrantes peuvent être exclues du processus de pilotage ;

3. la température sèche $\mathrm{T}\left({ }^{\circ} \mathrm{C}\right)$ qui doit régner dans la cellule. Sa régulation doit être suffisamment précise pour limiter l'amplitude des oscillations de température autour du point de consigne ;

4. I'humidité relative de l'air $\mathrm{Rh}(\%)$;

5. I'humidité d'équilibre du bois ( $\mathrm{H} \%$ éq.), aussi appelée équilibre hygroscopique du bois (en anglais EMC, Equilibrium moisture content; en allemand, $U G L, U$, symbole physique de I'humidité du bois, GL, Gleichgewicht), correspond au taux d'humidité vers lequel tend le bois placé dans des conditions climatiques stables (température et humidité relative de l'air).

Un séchoir à bois est piloté en fixant I'humidité d'équilibre du bois ou en fixant l'humidité relative de l'air. L'humidité d'équilibre du bois peut être déduite du couple de consignes $(\mathrm{Rh}, \mathrm{T})$ en utilisant les courbes d'équilibre hygroscopique du bois (courbes de Keylwerth) qui donnent $\mathrm{H} \%$ éq $=\mathrm{f}(\mathrm{Rh}, \mathrm{T})$. On utilise, pour ce faire, un programme de calcul ou directement un abaque qui reprend ces courbes.

Les neuf programmes de séchage proposés ont été élaborés en collaboration avec Monsieur Gérard Gandon (Olergie), en utilisant comme référence les programmes édités par Cathild Industrie.

Pour chaque essence, le programme de séchage est donné à titre indicatif pour des bois d'épaisseur inférieure à $35 \mathrm{~mm}$. Il est à valider par une mise en application dans le respect des règles de l'art. Pour des bois d'épaisseur comprise entre 35 et $55 \mathrm{~mm}$, il est conseillé d'augmenter l'humidité relative de l'air de $5 \%$ à chaque étape du séchage (par exemple, $30 \%$ pour une épaisseur de $27 \mathrm{~mm}$ et $35 \%$ pour une épaisseur de $41 \mathrm{~mm}$ ) et de diminuer la température de $3{ }^{\circ} \mathrm{C}$ à chaque étape du préchauffage, du séchage et de l'équilibrage.

Pour des bois d'épaisseur supérieure à $55 \mathrm{~mm}$, il est conseillé d'augmenter l'humidité relative de l'air de $10 \%$ à chaque étape du séchage et de diminuer la température de $5{ }^{\circ} \mathrm{C}$ à chaque étape du préchauffage, du séchage et de l'équilibrage.

\section{Programme de séchage 1}

\begin{tabular}{|l|c|c|c|c|c|}
\hline Phases & Durée (h) & H \% sondes & T $\left({ }^{\circ} \mathbf{C}\right)$ & Rh (\%) & UGL (\%) \\
\hline Préchauffage 1 & & $>50$ & 60 & 81 & 14,0 \\
\hline Préchauffage 2 & 3 & $>50$ & 65 & 76 & 12,0 \\
\hline Séchage & & $>50$ & 68 & 68 & 10,0 \\
& & $50-40$ & 70 & 63 & 9,1 \\
\hline & $40-35$ & 70 & 61 & 8,7 \\
\hline & $35-30$ & 70 & 56 & 7,9 \\
\hline & $30-27$ & 72 & 50 & 7,0 \\
\hline & $27-24$ & 72 & 44 & 6,3 \\
\hline & $24-21$ & 75 & 39 & 5,5 \\
\hline & $21-18$ & 75 & 34 & 4,9 \\
\hline & $18-15$ & 75 & 29 & 4,3 \\
\hline & $15-12$ & 80 & 28 & 3,9 \\
\hline & & $12-09$ & 80 & 24 & 3,4 \\
\hline & & $09-06$ & 80 & 22 & 3,2 \\
\hline
\end{tabular}

(1) Refroidissement : aussi longtemps que la température dans la cellule dépasse la température extérieure de plus de $30^{\circ} \mathrm{C}$.

(2) $U G L=H \%$ final $\times 0,8$ à 0,9 .

(3) Rh à déduire de I'UGL défini au (2) et de la température, sur les courbes de Keylwerth. 
Programme de séchage 2

\begin{tabular}{|c|c|c|c|c|c|}
\hline Phases & Durée (h) & H\% sondes & $\mathrm{T}\left({ }^{\circ} \mathrm{C}\right)$ & Rh (\%) & UGL (\%) \\
\hline Préchauffage 1 & & $>50$ & 58 & 84 & 15,0 \\
\hline Préchauffage 2 & 3 & $>50$ & 63 & 81 & 13,5 \\
\hline \multirow[t]{12}{*}{ Séchage } & & $>50$ & 65 & 72 & 11,0 \\
\hline & & $50-40$ & 68 & 68 & 10,1 \\
\hline & & $40-35$ & 68 & 62 & 9,0 \\
\hline & & $35-30$ & 70 & 60 & 8,5 \\
\hline & & $30-27$ & 72 & 54 & 7,6 \\
\hline & & $27-24$ & 72 & 50 & 7,0 \\
\hline & & $24-21$ & 74 & 43 & 6,1 \\
\hline & & $21-18$ & 74 & 36 & 5,2 \\
\hline & & $18-15$ & 75 & 31 & 4,5 \\
\hline & & $15-12$ & 75 & 28 & 4,2 \\
\hline & & $12-09$ & 75 & 25 & 3,8 \\
\hline & & $09-06$ & 75 & 24 & 3,6 \\
\hline Équilibrage & 6 & & 68 & (3) & (2) \\
\hline Refroidissement & (1) & & Arrêt & (3) & (2) \\
\hline
\end{tabular}

(1) Refroidissement : aussi longtemps que la température dans la cellule dépasse la température extérieure de plus de $30^{\circ} \mathrm{C}$.

(2) $U G L=H \%$ final $\times 0,8$ à 0,9 .

(3) Rh à déduire de I'UGL défini au (2) et de la température, sur les courbes de Key/werth.

\section{Programme de séchage 3}

\begin{tabular}{|l|c|c|c|c|c|}
\hline Phases & Durée (h) & H \% sondes & T $\left({ }^{\circ} \mathbf{C}\right)$ & Rh (\%) & UGL (\%) \\
\hline Préchauffage 1 & & $>50$ & 55 & 84 & 15,5 \\
\hline Préchauffage 2 & 3 & $>50$ & 57 & 83 & 15,0 \\
\hline Séchage & & $>50$ & 60 & 76 & 12,5 \\
\hline & & $50-40$ & 60 & 73 & 11,6 \\
\hline & & $40-35$ & 60 & 69 & 10,7 \\
\hline & & $35-30$ & 60 & 62 & 9,5 \\
\hline & & $30-27$ & 63 & 55 & 8,2 \\
\hline & & $27-24$ & 64 & 50 & 7,5 \\
\hline & & $21-18$ & 65 & 46 & 6,9 \\
\hline & & $18-15$ & 65 & 39 & 6,0 \\
\hline
\end{tabular}

(1) Refroidissement : aussi longtemps que la température dans la cellule dépasse la température extérieure de plus de $30^{\circ} \mathrm{C}$.

(2) $U G L=H \%$ final $\times 0,8$ à 0,9 .

(3) Rh à déduire de I'UGL défini au (2) et de la température, sur les courbes de Keylwerth. 
Programme de séchage 4

\begin{tabular}{|c|c|c|c|c|c|}
\hline Phases & Durée (h) & H\% sondes & $\mathbf{T}\left({ }^{\circ} \mathrm{C}\right)$ & Rh (\%) & UGL (\%) \\
\hline Préchauffage 1 & & $>50$ & 50 & 86 & 16,5 \\
\hline Préchauffage 2 & 3 & $>50$ & 52 & 85 & 16,0 \\
\hline \multirow[t]{12}{*}{ Séchage } & & $>50$ & 55 & 82 & 14,7 \\
\hline & & $50-40$ & 55 & 80 & 13,8 \\
\hline & & $40-35$ & 55 & 75 & 12,6 \\
\hline & & $35-30$ & 56 & 73 & 12,0 \\
\hline & & $30-27$ & 58 & 67 & 10,5 \\
\hline & & $27-24$ & 60 & 58 & 8,9 \\
\hline & & $24-21$ & 62 & 50 & 7,5 \\
\hline & & $21-18$ & 64 & 45 & 6,8 \\
\hline & & $18-15$ & 65 & 37 & 5,7 \\
\hline & & $15-12$ & 65 & 34 & 5,3 \\
\hline & & $12-09$ & 65 & 28 & 4,5 \\
\hline & & $09-06$ & 65 & 24 & 4,0 \\
\hline Équilibrage & 6 & & 58 & (3) & (2) \\
\hline Refroidissement & (1) & & Arrêt & (3) & (2) \\
\hline
\end{tabular}

(1) refroidissement : aussi longtemps que la température dans la cellule dépasse la température extérieure de plus de $30^{\circ} \mathrm{C}$.

(2) $U G L=H \%$ final $\times 0,8$ à 0,9 .

(3) Rh à déduire de I'UGL défini au (2) et de la température, sur les courbes de Keylwerth.

\section{Programme de séchage 5}

\begin{tabular}{|c|c|c|c|c|c|}
\hline Phases & Durée $(\mathrm{H})$ & H\% Sondes & $\mathrm{T}\left({ }^{\circ} \mathrm{C}\right)$ & Rh (\%) & UGL (\%) \\
\hline Préchauffage 1 & & $>50$ & 50 & 87 & 17,0 \\
\hline Préchauffage 2 & 4 & $>50$ & 50 & 86 & 16,5 \\
\hline \multirow[t]{12}{*}{ Séchage } & & $>50$ & 53 & 83 & 15,2 \\
\hline & & $50-40$ & 53 & 80 & 14,1 \\
\hline & & $40-35$ & 54 & 80 & 13,9 \\
\hline & & $35-30$ & 55 & 75 & 12,5 \\
\hline & & $30-27$ & 57 & 70 & 11,0 \\
\hline & & $27-24$ & 58 & 61 & 9,4 \\
\hline & & $24-21$ & 59 & 51 & 7,9 \\
\hline & & $21-18$ & 60 & 47 & 7,3 \\
\hline & & $18-15$ & 61 & 39 & 6,1 \\
\hline & & $15-12$ & 62 & 35 & 5,6 \\
\hline & & $12-09$ & 62 & 30 & 5,0 \\
\hline & & $09-06$ & 62 & 26 & 4,4 \\
\hline Équilibrage & 8 & & 55 & (3) & (2) \\
\hline Refroidissement & (1) & & Arrêt & (3) & (2) \\
\hline
\end{tabular}

(1) Refroidissement : aussi longtemps que la température dans la cellule dépasse la température extérieure de plus de $30^{\circ} \mathrm{C}$.

(2) $U G L=H \%$ final $\times 0,8$ à 0,9 .

(3) Rh à déduire de I'UGL défini au (2) et de la température, sur les courbes de Keylwerth. 
Programme de séchage 6

\begin{tabular}{|c|c|c|c|c|c|}
\hline Phases & Durée (H) & H\% Sondes & $\mathrm{T}\left({ }^{\circ} \mathrm{C}\right)$ & Rh (\%) & UGL (\%) \\
\hline Préchauffage 1 & & $>50$ & 50 & 87 & 17,0 \\
\hline Préchauffage 2 & 4 & $>50$ & 50 & 86 & 16,5 \\
\hline \multirow[t]{12}{*}{ Séchage } & & $>50$ & 53 & 85 & 15,7 \\
\hline & & $50-40$ & 53 & 82 & 14,6 \\
\hline & & $40-35$ & 54 & 78 & 13,4 \\
\hline & & $35-30$ & 55 & 77 & 12,9 \\
\hline & & $30-27$ & 57 & 73 & 11,9 \\
\hline & & $27-24$ & 58 & 68 & 10,7 \\
\hline & & $24-21$ & 60 & 61 & 9,3 \\
\hline & & $21-18$ & 62 & 52 & 7,9 \\
\hline & & $18-15$ & 64 & 43 & 6,6 \\
\hline & & $15-12$ & 65 & 39 & 6,0 \\
\hline & & $12-09$ & 65 & 31 & 5,0 \\
\hline & & $09-06$ & 65 & 28 & 4,5 \\
\hline Équilibrage & 8 & & 58 & (3) & (2) \\
\hline Refroidissement & (1) & & Arrêt & (3) & (2) \\
\hline
\end{tabular}

(1) Refroidissement : aussi longtemps que la température dans la cellule dépasse la température extérieure de plus de $30^{\circ} \mathrm{C}$.

(2) $U G L=H \%$ final $\times 0,8$ à 0,9 .

(3) Rh à déduire de I'UGL défini au (2) et de la température, sur les courbes de Key/werth.

\section{Programme de séchage 7}

\begin{tabular}{|l|c|c|c|c|c|}
\hline Phases & Durée (H) & $\mathbf{H} \%$ Sondes & T $\left({ }^{\circ} \mathbf{C}\right)$ & Rh (\%) & UGL (\%) \\
\hline Préchauffage 1 & & $>50$ & 40 & 86 & 17,0 \\
\hline Préchauffage 2 & 4 & $>50$ & 43 & 85 & 16,5 \\
\hline Séchage & & $>50$ & 45 & 83 & 15,7 \\
\hline & & $50-40$ & 45 & 80 & 14,6 \\
\hline & & $40-35$ & 45 & 77 & 13,8 \\
\hline & & $35-30$ & 45 & 74 & 12,9 \\
\hline & & $27-27$ & 47 & 69 & 11,5 \\
\hline & & $24-21$ & 49 & 61 & 9,9 \\
\hline & & $21-18$ & 50 & 52 & 8,4 \\
\hline & & $18-15$ & 56 & 48 & 7,7 \\
\hline
\end{tabular}

(1) Refroidissement : aussi longtemps que la température dans la cellule dépasse la température extérieure de plus de $30^{\circ} \mathrm{C}$.

(2) $U G L=H \%$ final $\times 0,8$ à 0,9 .

(3) Rh à déduire de I'UGL défini au (2) et de la température, sur les courbes de Keylwerth. 
Programme de séchage 8

\begin{tabular}{|c|c|c|c|c|c|}
\hline Phases & Durée (H) & H\% Sondes & $\mathrm{T}\left({ }^{\circ} \mathrm{C}\right)$ & Rh (\%) & UGL (\%) \\
\hline Préchauffage 1 & & $>50$ & 45 & 86 & 17,0 \\
\hline Préchauffage 2 & 4 & $>50$ & 45 & 85 & 16,5 \\
\hline \multirow[t]{12}{*}{ Séchage } & & $>50$ & 48 & 84 & 15,7 \\
\hline & & $50-40$ & 48 & 80,5 & 14,6 \\
\hline & & $40-35$ & 49 & 77 & 13,4 \\
\hline & & $35-30$ & 50 & 75 & 12,9 \\
\hline & & $30-27$ & 51 & 70 & 11,5 \\
\hline & & $27-24$ & 53 & 62 & 9,9 \\
\hline & & $24-21$ & 54 & 53 & 8,4 \\
\hline & & $21-18$ & 55 & 48,5 & 7,7 \\
\hline & & $18-15$ & 55 & 40 & 6,6 \\
\hline & & $15-12$ & 55 & 35 & 5,9 \\
\hline & & $12-09$ & 60 & 30 & 5,0 \\
\hline & & $09-06$ & 60 & 28 & 4,7 \\
\hline Équilibrage & 8 & & 58 & (3) & (2) \\
\hline Refroidissement & (1) & & Arrêt & (3) & (2) \\
\hline
\end{tabular}

(1) Refroidissement : aussi longtemps que la température dans la cellule dépasse la température extérieure de plus de $30^{\circ} \mathrm{C}$.

(2) $U G L=H \%$ final $\times 0,8$ à 0,9 .

(3) Rh à déduire de I'UGL défini au (2) et de la température, sur les courbes de Keylwerth.

\section{Programme de séchage 9}

\begin{tabular}{|l|c|c|c|c|c|}
\hline Phases & Durée (H) & H\% Sondes & T $\left({ }^{\circ} \mathbf{C}\right)$ & Rh (\%) & UGL (\%) \\
\hline Préchauffage 1 & & $>40$ & 35 & 87 & 18,0 \\
\hline Préchauffage 2 & 6 & $>40$ & 38 & 85 & 17,0 \\
\hline Séchage & & $>40$ & 41 & 82 & 15,7 \\
\hline & & $40-35$ & 44 & 81 & 15,0 \\
\hline & $35-30$ & 46 & 80 & 14,5 \\
\hline & & $30-25$ & 48 & 77 & 13,5 \\
\hline & & $25-20$ & 50 & 72 & 12,0 \\
\hline & & $18-18$ & 52 & 63 & 10,0 \\
\hline & & $16-14$ & 54 & 54 & 8,5 \\
\hline
\end{tabular}

(1) Refroidissement : aussi longtemps que la température dans la cellule dépasse la température extérieure de plus de $30^{\circ} \mathrm{C}$.

(2) $U G L=H \%$ final $\times 0,8$ à 0,9 .

(3) Rh à déduire de I'UGL défini au (2) et de la température, sur les courbes de Key/werth. 


\section{Sciage et usinage}

\section{Effet désaffûtant}

Cet effet est essentiellement lié au taux de silice du bois et à sa dureté ; il conditionne le type d'outils utilisés lors du sciage et de l'usinage.

\section{Denture pour le sciage}

Selon l'effet désaffûtant du bois, il est recommandé d'utiliser des dentures en acier ordinaire ou en acier allié, ou des dentures stellitées.

\section{Outils d'usinage}

Le choix de l'outillage est lié à l'abrasivité du bois, c'est-à-dire à son effet désaffûtant. Si cet effet désaffûtant est normal, un outillage normal est conseillé. Dans le cas contraire, des outils spéciaux, en acier rapide type HSS ou au carbure de tungstène, sont préconisés.

Certains bois contiennent des substances chimiques qui peuvent leur conférer, à des degrés divers, une certaine toxicité auprès de certains utilisateurs lors de la transformation (dégagement de poussières lors du sciage et de l'usinage) et provoquer des allergies, des irritations de la peau ou des muqueuses et, dans les cas extrêmes, des troubles respiratoires. Le port d'équipements individuels de protection (EPI) et l'utilisation de systèmes efficaces d'aspiration des poussières (obligations légales) permettent d'éviter ou de limiter ces risques.

\section{Aptitude au déroulage}

Les essences habituellement recherchées en déroulage sont tendres à mi-dures. Les grumes doivent être bien conformées et sans défaut afin d'obtenir un bon rendement. De plus, le bois doit avoir un bon comportement au séchage afin de limiter les risques de fentes et de déformations des placages.

\section{Aptitude au tranchage}

Les bois recherchés doivent présenter des qualités esthétiques bien définies : couleur, figuration, finesse du grain et aspect rubané.

\section{Assemblage}

\section{Clouage et vissage}

Il est important de savoir si l'on peut enfoncer facilement une pointe ou une vis dans le bois sans risque de fente. Dans le cas contraire, la nécessité de réaliser des avant-trous est précisée.

De plus, l'aptitude d'un bois à retenir une pointe ou une vis est mentionnée : bonne ou mauvaise tenue.

\section{Collage}

Dans l'industrie du bois, le secteur du collage, notamment le collage des bois tropicaux, est sans doute celui qui a le plus progressé depuis les années 1980. La mise sur le marché de nouveaux adhésifs toujours plus performants permet de coller tous les bois sans restriction, quelles que soient leurs caractéristiques, avec des exigences accrues de résistance à l'eau et de résistance mécanique.

Ces avancées technologiques permettent d'optimiser l'utilisation des bois tropicaux grâce au collage de débits obtenus à partir d'essences secondaires, de grumes mal conformées ou de petit diamètre, de bois présentant des défauts importants, de bois déclassés et de déchets de scierie.

Les produits tels que le bois massif reconstitué (BMR) et les carrelets lamellés collés sont stables et homogènes. L'association par collage d'essences d'aspects très différents offre des perspectives pour intégrer davantage le bois dans des réalisations haut de gamme. Le collage du bois sur d'autres matériaux est possible.

Cependant, le collage peut être limité par des contraintes liées aux caractéristiques de certaines essences et à la nécessité de respecter les règles de l'art. C'est notamment le cas des essences à densité élevée décrites dans cet ouvrage (densité moyenne supérieure à 0,80). 
Des études menées notamment au Cirad ont permis de démontrer que le collage de bois présentant des défauts marqués ou des caractéristiques a priori défavorables peut donner des résultats satisfaisants. Cependant, il faut que les conditions de mise en œuvre préconisées soient respectées. Le choix de l'adhésif est fonction de l'emploi final du produit collé, du système de production, du temps d'assemblage nécessaire et du temps de pressage souhaité.

\section{Classements commerciaux}

\section{Classement d'aspect des sciages tempérés}

\section{Principe}

Les critères généralement retenus pour classer les bois sont la présence et l'importance de défauts : nœuds, fentes, poches de résine, pente de fil, flaches, altérations biologiques, déformations géométriques... Ces défauts sont susceptibles d'affecter les caractéristiques esthétiques des bois et de diminuer le volume de bois réellement utilisable. Certains défauts peuvent être acceptés. Cependant, ils entraînent une réduction de la surface ou du volume du bois servant de base au calcul du prix (plots), ou un déclassement sans modification de surface ou de volume (avivés).

\section{Règles de classement}

La norme NF EN 975-1 (avril 2009) décrit le classement d'aspect des bois sciés de Chêne européen et de Hêtre européen. La norme NF EN 975-2 (novembre 2004) concerne les bois sciés de Peuplier.

La norme NF EN 1611-1 (octobre 1999) concerne les bois sciés européens d'Epicéa, Sapin, Pin et Douglas. Dans la norme NF EN 1611-1/A1 (mars 2003), le Mélèze européen est ajouté à cette liste sans modifier les critères de classement.

\section{Cas particulier du Western red cedar nord-américain}

Éditée par le Pacific Lumber Inspection Bureau (PLIB, 2003), I'Export R-List Grading and Dressing Rules présente les règles de classement américaines (États-Unis) concernant les essences suivantes : Douglas fir, Pacific coast (West coast) Hemlock \& True firs, Sitka spruce et Red cedar.

Les règles de classement canadiennes NLGA (National Lumber Grades Authority) concernent les bois d'œuvre canadiens et notamment le Red cedar (janvier 2008).

\section{Classement d'aspect des sciages tropicaux}

\section{Principe}

Pour les bois tropicaux, deux modes de classement d'aspect des sciages sont utilisés :

- le classement de la pièce considérée selon le nombre de défauts standards présents en fonction de ses dimensions. En effet, plus la surface de la pièce est grande, plus le nombre de défauts tolérés est élevé. C'est le principe de classement actuel des sciages avivés africains (règles ATIBT, édition 1999). Ce classement est remplacé progressivement au profit des règles SATA (Sciages avivés tropicaux africains) ;

- le classement par découpe nette. Il définit le pourcentage de surfaces rectangulaires nettes de défaut par rapport à la surface de la pièce. Le choix de la pièce est défini par le pourcentage de défauts.

Pour les règles SATA, une pièce de choix I doit présenter $90 \%$ de surface nette de défauts. Une pièce de choix II doit présenter $80 \%$ de surface nette de défauts, ainsi de suite, avec un nombre de découpes variable par rapport à la surface de la pièce.

Ce principe est utilisé par les Malaysian Grading Rules (MGR) et dans les règles de la National Hardwood Lumber Association (NHLA).

\section{Règles de classement}

Les règles de classement SATA ont été élaborées en 1976 sous l'égide de la Commission européenne, à la demande de cinq États africains (Cameroun, Congo, Côte d'Ivoire, Gabon et 
République démocratique du Congo). Cette étude a été conduite par le Centre technique forestier tropical (CTFT, Nogent-sur-Marne). Ces règles ont été rééditées en 1996 dans l'ouvrage "Sciages avivés tropicaux africains - Règles de classement ».

Les règles de classement NHLA ont été établies à l'origine pour les feuillus nord-américains. La version actuelle (2015) concerne également certaines essences tropicales : "Rules for the Measurement \& Inspection of Hardwood \& Cypress ». Elle est disponible sur le site internet de la NHLA.

Les règles « Bois guyanais classés » (1990) ont été établies pour les bois exploités et transformés en Guyane française. Elles sont essentiellement utilisées sur le marché guyanais ou les exportations à destination des Antilles.

Les règles de classement MGR ont été établies en 1968, révisées en 1984 puis en juillet 2009. Elles concernent les essences de Malaisie, mais elles peuvent être utilisées pour des bois d'autres provenances asiatiques.

\section{Classement visuel de structure}

Dans cet ouvrage, le classement visuel de structure des bois tropicaux et des bois feuillus se réfère d'une part aux normes françaises NF B 52-001-1 (août 2011), NF B 52-001-1/A1 (avril 2013) et NF B 52-001/A2 (février 2015) et d'autre part, à la norme européenne EN 1912 (2012) associée aux normes nationales correspondantes. Dans cet ouvrage, 105 essences décrites font l'objet d'un classement mécanique qui peut être attribué par classement visuel conformément aux règles définies dans une ou plusieurs de ces normes.

\section{Réaction au feu}

Deux propriétés différentes du bois caractérisent son comportement au feu : la résistance au feu et la réaction au feu.

La résistance au feu est la durée pendant laquelle un matériau soumis à un feu peut continuer d'assurer ses fonctions initiales dans l'ouvrage : il s'agit de la stabilité mécanique de la structure, la fonction de coupe-feu ou de pare-flamme d'une porte, etc.

La réaction au feu caractérise la production de flammes, de chaleur, de fumées, de particules et de gouttes du matériau soumis au feu. La réaction au feu concerne essentiellement les matériaux utilisés dans les revêtements de sol, murs et plafonds qui sont en contact avec les personnes.

Les informations contenues dans cet ouvrage concernent la réaction au feu.

\section{Classement conventionnel français}

La réglementation française (classement conventionnel, arrêté du 21 novembre 2002) définit six classes de réaction au feu :

- MO - incombustibles ;

- M1 - non inflammables;

- M2 - difficilement inflammables;

- M3 - moyennement inflammables;

- M4 - facilement inflammables ;

- M5 - très facilement inflammables.

D’une manière générale, le classement des bois massifs non traités est le suivant.

Bois massif non résineux :

- épaisseurs supérieures ou égales à 14 mm : M3 - moyennement inflammables ;

- épaisseurs inférieures à 14 mm : M4 - facilement inflammables.

Bois massif résineux :

- épaisseurs supérieures ou égales à 18 mm : M3 - moyennement inflammables ;

- épaisseurs inférieures à $18 \mathrm{~mm}$ : M4 - facilement inflammables. 


\section{Classement selon les euroclasses}

La normalisation européenne (euroclasses de la NF EN 13501-1+A1, février 2013) définit une nouvelle classification fondée sur des méthodes d'essai différentes :

- A1, A2, B, C, D, E et F pour les revêtements de murs et de plafonds ;

- $A 1_{f f}, A 2_{f l}, B_{f \mid}, C_{f l}, D_{f \mid}, E_{f l}$ et $F_{f l}$ pour les revêtements de sol.

Les informations contenues dans cet ouvrage concernent les revêtements de murs et de plafonds.

À l'intérieur de chaque classe, deux critères de réaction au feu supplémentaires caractérisent, d'une part, la production de fumées $(\mathrm{s} 0, \mathrm{~s} 1, \mathrm{~s} 2)$ et, d'autre part, la production de gouttes et de particules incandescentes $(\mathrm{d} 0, \mathrm{~d} 1, \mathrm{~d} 2)$.

En considérant les propriétés des bois les plus couramment utilisés en Europe (NF EN 14081-1, avril 2016), le bois massif est par convention classé « D-s2, d0 » en revêtement de mur et de plafond si sa densité est supérieure à 0,35 . Si la densité du bois est inférieure à 0,35 , l'essence est considérée comme hors classement, c'est le cas des essences suivantes : Balsa, Emien, Essessang, Fromager, Pashaco et Sumauma.

Tout autre classement plus favorable doit être démontré par essai. Pour les essences décrites dans cet Atlas, c'est le cas du Mélèze et des essences tropicales d'Amérique latine et de la Guyane : Mandioqueira, Pau roxo, Basralocus, Ipê et Louro vermelho.

\section{Principales utilisations}

Les possibilités d'emploi d'un bois dépendent directement de ses propriétés technologiques. La liste des utilisations mentionnées est non exhaustive. Elle correspond aux principales utilisations connues qui doivent être validées par une mise en œuvre respectant les règles de I'art. Certaines utilisations sont mentionnées à titre d'information (utilisations traditionnelles, régionales ou anciennes).

\section{Principales appellations vernaculaires}

Les principaux noms vernaculaires en usage dans les pays producteurs sont mentionnés, ainsi que les noms commerciaux en usage dans les pays importateurs quand ils sont différents du nom pilote ATIBT.

Pour chaque essence décrite, la liste des «Principales appellations vernaculaires » n'est pas exhaustive. Résultant d'une compilation très complète, un inventaire quasi exhaustif de ces appellations vernaculaires est proposé sur le site du Forest Products Laboratory de Madison (États-Unis). www.fpl.fs.fed.us/search/commonname_request.php. 


\section{Addendum extract from Tropical Timber Atlas User guide: general information}

\section{Names and Commercial Restrictions (CITES)}

\section{Common names for species}

The common names are those referenced in the Nomenclature Générale des Bois Tropicaux published by the Association Technique Internationale des Boix Tropicaux (ATIBT) in 2016. This reference guide is internationally recognised, particularly in Europe, with the implementation of the European Union Timber Regulation (EUTR).

For certain species, the common name, which is the term most referred to, is accompanied by a second, frequently used trade name, indicated by an asterisk. For instance, the following species have been identified with two names: Alan / Alan-Batu*; Anzèm / Nténé*; Balau, Yellow / Bangkirai*; Catucaém / Louro Faia*; Coraçao de negro* / Panacoco; Cryptomeria* / Sugi; Dukali / Amapa*; Fuma / Fromager*; Kurokaï / Breu*; Mango / Machang*; Pashaco / Paricá*; Pinus kesiya* / Kesiya Pine; Pinus merkusii* / Merkusii Pine; Pinus patula* / Patula Pine.

\section{Family and botanical names}

Like the common names, the family and botanical names listed are also referenced in the Nomenclature Générale des Bois Tropicaux (ATIBT, 2016).

The following abbreviations appear after certain botanical names:

\footnotetext{
- "spp." (species pluralis) and "p.p." (pro parte). In botany, the abbreviation "spp." means 'more than one species in the genus'. It can include all species in a given genus, which can be confusing. Different authors use this abbreviation differently, to designate several species within a genus in a non-exhaustive manner, or to designate all species in that genus.

- In this Atlas, the abbreviation "p.p." is used for types of wood which include several - but not every - species within a genus;

- "subgen." (subgenus). In a given genus, several significantly similar species can form a subgenus. In a subgenus, a wood type can cover all species, several species, or simply some of these, as per the conditions mentioned above.
}

\section{CITES (Washington Convention of 2017)}

CITES (the Convention on International in Endangered Species of Wild Fauna and Flora, or 'the Washington Convention') is an international agreement between governments. Its aim is to ensure that international trade in specimens of wild animals and plants does not threaten their survival. Tropical wood is therefore protected by this convention.

Regulated wood species are classified in one of the convention's three appendices:

- Appendix I includes species threatened with extinction. Trade in specimens of these species is permitted only in exceptional circumstances;

- Appendix II includes species not necessarily threatened with extinction, but in which trade must be controlled in order to avoid use incompatible with their survival;

- Appendix III contains species that are protected in at least one country. This country must ask other CITES parties for assistance in controlling the trade. Changes to Appendix III follow a distinct procedure from changes to Appendices I and II, as each party is entitled to make unilateral amendments to it.

The label "no trade restrictions" is applied to species not listed in CITES. For more information, consult the CITES web site: www.cites.org. 


\section{Log description}

\section{Diameter}

The range of mentioned values corresponds to the diameters of the most frequently exploited woods. These values must be weighed against $\mathrm{MDCL}$ - minimum-diameter cutting limits determined by each producer country to ensure acceptable forest sustainability after the rotation period. MDCL values are generally available from the forestry services of these countries.

\section{Thickness of sapwood}

The range of mentioned values corresponds to the most frequently encountered thicknesses of sapwood.

\section{Buoyancy}

Two classes (floatable and non-floatable woods) were defined according to the average density of green woods (after felling). A third class ('not applicable') was established for species in temperate countries.

\section{Log conservation}

Depending on the wood's natural durability, preservation is low (the wood must be treated), moderate (treatment recommended) or good. The concept of preservation only applies to heartwood; sapwood is always considered as non-durable.

\section{Wood description}

\section{Colour}

Although the colour and appearance of the wood are usually specific to a given species, the colour is not a constant factor from one tree to another or from one piece of wood to another of the same species. It can vary according to different parameters and change over time. Variations in grain gradient (for example, interlocked grain and wavy grain) and moisture content can alter the perception of colour.

Each species is characterised by a reference colour chosen from among 18 predefined colours: white, cream white, pinkish white, light yellow, yellow, orange yellow, light brown, brown, yellow brown, pinkish brown, red brown, dark brown, light red, red, dark red, grey, black and purple.

The description of the wood colour mentioned in the note under "Wood description" emphasises the range of variation found, but does not take into account, for example, the change in the colour of woods exposed to bad weather.

\section{Sapwood}

The sapwood can be well-demarcated, poorly demarcated or not demarcated. The rating "n.d." is used in cases where no information is available.

\section{Texture}

The texture of a wood corresponds to the visual impression given by the size and the arrangement of the vessels. Three classes of texture are defined: fine, medium and coarse.

\section{Grain and interlocked grain}

The grain of the wood is the general alignment of the fibres in relation to the log. The interlocked grain is due to an alternate incline (in relation to the trunk axis) of the successive layers of the wood that form during tree growth.

\section{Physical and mechanical properties}

The values of the physical and mechanical properties (mean values) are computed from tests conducted at CIRAD laboratories or obtained from international literature. They must be used with caution due to the highly variable nature of wood properties. This variability is wellknown by people working in the wood industry. It depends on numerous external or internal factors: the age of the trees, the position of the wood inside the trunk, wood maturity, and growth conditions (including soil type, rainfall and climate). 


\section{Density}

The density or relative density of a solid is the ratio of its mass per unit volume over the mass per unit volume of water (pure water at $4{ }^{\circ} \mathrm{C}$ at atmospheric pressure, i.e. $1,000 \mathrm{~kg} / \mathrm{m}^{3}$ ). It has no unit.

Indicated density is determined on wood at $12 \%$ moisture content. This basic technological characteristic is the first to be determined when qualifying wood. This property is more or less closely related to the wood's main physical and mechanical properties and with certain working characteristics.

\section{Monnin hardness}

Monnin hardness (determined on wood at $12 \%$ moisture content) is an important property to know when the wood is used for flooring (parquets, decking) or any end-use where the wood will be subject to impacts or punching. It has no unit.

Hardness classification:

- $\mathrm{H} \leq 1.5$ : very soft;

$1.5 \leq \mathrm{H} \leq 3$ : soft;

- $3 \leq \mathrm{H} \leq 6$ : medium;

- $6 \leq \mathrm{H} \leq 9$ : hard;

- $\mathrm{H} \leq$ 9: very hard;

The method of measurement of Monnin hardness is defined by the French NF B 51-013 standard (1985).

Janka hardness is another characteristic, measured in several countries, using another method. Sallenave (1971) suggests the following ratio between Monnin hardness and Janka hardness: Janka hardness (in pounds) $=300 \times$ Monnin hardness.

Fibre saturation point (FSP, in \%)

In green wood, part of the water fills, more or less completely, the cellular and intercellular empty spaces. The draining of this free water occurs without wood shrinkage. Once free water has completely disappeared, the wood only contains bound water impregnating the cell walls. When this bound water evaporates during drying, shrinkage occurs and provokes wood warping.

The fibre saturation point (FSP) corresponds to the moisture content of wood saturated with bound water. Below this threshold, the wood starts to shrink during drying. The FSP usually varies between 20 and $40 \%$ according to species, but most often, it is around $30 \%$.

Fibre saturation point classification:

- FSP $\leq 25 \%$ : low;

- $25 \% \leq \mathrm{FSP} \leq 35 \%$ : medium;

- FSP $\geq 35 \%$ : high.

\section{Coefficient of volumetric shrinkage (Vs, in \% by \%)}

When a piece of wood dries below its fibre saturation point (FSP), its volume decreases. If it reabsorbs moisture, its volume increases up to the FSP. Above that, the volume no longer varies. In order to quantify these volume variations, the coefficient of volumetric shrinkage is used (called Vs) and corresponds to the volumetric shrinkage of a piece of wood when its moisture content has a variation of $1 \%$.

Classification for the coefficient of volumetric shrinkage:

- Vs $\leq$ 0.35: small shrinkage;

$0.35 \leq \mathrm{Vs} \leq 0.55$ : medium shrinkage;

- Vs $\leq$ 0.55: large shrinkage. 
Until the fibre saturation point, the wood does not shrink during drying. Once below this threshold, however, it is subject to dimensional variations when its moisture content varies. Shrinkage under the FSP occurs in the wood's three directions: longitudinal, tangential and radial.

Longitudinal shrinkage is very small compared to the two others, about some tenths of a percent, but it can notably influence the dimensional variations of long wood pieces. Few data are available on this characteristic which is quite difficult to measure in the laboratory.

Total tangential and total radial shrinkages are usually determined to qualify the behaviour of wood during drying or more generally during moisture variations.

Total tangential shrinkage classification (Ts):

- Ts $\leq 6.5 \%$ : small shrinkage;

- $6.5 \% \leq$ Ts $\leq 10 \%$ : medium shrinkage;

- Ts $\geq 10 \%$ : large shrinkage.

Total radial shrinkage classification (Rs):

- Rs $\leq 3.8 \%$ : small shrinkage;

- $3.8 \% \leq$ Rs $\leq 6.5 \%$ : medium shrinkage;

- Rs $\geq 6.5 \%$ : large shrinkage.

\section{Ts/Rs ratio}

The "Total tangential shrinkage" over "Total radial shrinkage" ratio gives an indication of the deformation suffered by a piece of wood subjected to humidity variations.

This parameter is of particular importance for non-directional cuts (half-quartered cuts). A Ts/Rs ratio tending towards a value greater than or equal to 2 indicates that a species is susceptible to deformation. The more this value tends towards 1 , the more stable the wood, whatever the type of cut.

\section{Thermal conductivity}

The thermal conductivity $\lambda$ (watt per metre and per Kelvin: $\mathrm{W} / \mathrm{m} \cdot \mathrm{K}$ ) of a substance is its ability to conduct heat. The more insulating a substance is, the lower the $\lambda$.

The $\lambda$ values specified in this atlas for each wood species are the result of a campaign to measure a broad sample of tropical and temperate woods of a wide range of densities. However this sample does not cover all the species included in the atlas.

Measurements were made in the thermal physics lab of the Heterogeneous Materials Study Group (GEMH) at the European Ceramics Centre in Limoges, France) using the hot disk method regulated by the NF EN ISO 22007-2 standard (October 2015). This measurement campaign revealed that thermal conductivity $(\lambda)$ is correlated with wood density (D) (Figure 1).

Thermal conductivity $\lambda$ in relation to density $D$ is therefore expressed in the following equation: $\lambda=0,289 \mathrm{D}+0,030$.

The $\lambda$ values specified in this atlas were determined using this model, based on the average density of each wood species.

\section{Heating value}

The heating value (or calorific value) of a substance is defined as the amount of heat released by its combustion. It is usually measured in kilojoules per kilogram $(\mathrm{kJ} / \mathrm{kg})$ or joules per gram $\mathrm{J} / \mathrm{g}$ ), sometimes in calories per gram (cal/g) or kilocalories per kilogram (kcal/kg). Two types of heating value can be defined:

- The higher heating value (HHV) is the amount of heat released by combustion, at constant volume, of $1 \mathrm{~kg}$ of an anhydrous substance. Water produced during combustion is condensed, while the heat released by water condensation (latent heat of vaporisation) is recovered; 


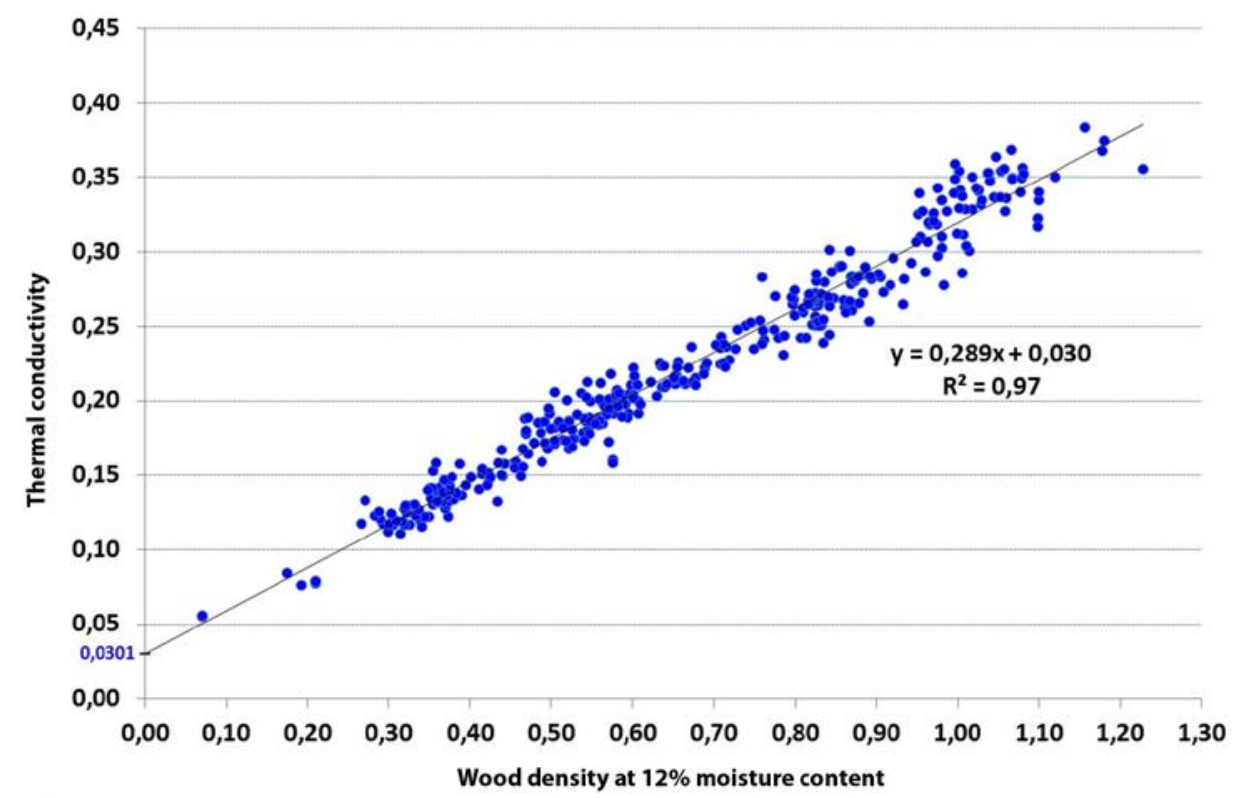

Figure 1 . Thermal conductivity in relation to wood density (determined at $12 \%$ moisture content).

- The lower heating value (LHV) is the amount of heat released by combustion, at constant pressure, in open air, of $1 \mathrm{~kg}$ of an anhydrous substance. Water produced during combustion is not condensed. Therefore, energy produced by condensation is not recovered.

LHV is the value most commonly used when calculating combustion. It is measured in $\mathrm{kJ} / \mathrm{kg}$ of anhydrous wood.

HHV is defined in an experimental (lab) setting using an object called a "bomb calorimeter". For wood, the lower heating value ( $\mathrm{LHV}$, in $\mathrm{kJ} / \mathrm{kg}$ ) is subtracted from the higher heating value (HHV) using the following equation:

- Anhydrous LHV = Anhydrous HHV - $(212.2 \times \mathrm{H})$

where $\mathrm{H}$ is the hydrogen content (expressed in \% by weight) of a given biomass model regulated by the "Solid biofuels - Determination of calorific value" NF EN 14918 standard (March 2010).

The LHV specified in this atlas were determined using HHV values measured in a laboratory at CIRAD. Hydrogen $\mathrm{H}$ content is not determined during the experiment. Therefore, an average $\mathrm{H}$ content of $5.85 \%$ was used for the calculation (experience shows that the $\mathrm{H}$ value is nearly identical from one species to another).

Crushing strength (in $\mathrm{MPa}$ )

This resistance (called $\mathrm{C}_{12}$ ) is determined on wood at $12 \%$ moisture content, according to the procedure stipulated in NF B 51-007 standard (September 1985). It corresponds to the load to be applied parallel to the grain to achieve rupture of a standardized sample.

Crushing strength classification:

- $\mathrm{C} 12 \leq 45 \mathrm{MPa}$ : low resistance;

- $45 \mathrm{MPa} \leq \mathrm{C} 12 \leq 75 \mathrm{MPa}$ : moderate resistance;

- $\mathrm{C} 12 \geq 75$ MPa: high resistance. 


\section{Static bending strength (in MPa)}

The static bending strength (called $B_{12}$ ) is determined on wood at $12 \%$ moisture content, according to the procedure stipulated in standard B 51-008 (November 1987). It corresponds to the load to be applied to the middle of a standardized sample placed between two supports to achieve rupture.

Static bending strength classification:

- F12 $\leq 75$ MPa: low resistance;

- $75 \mathrm{MPa} \leq \mathrm{F} 12 \leq 125 \mathrm{MPa}$ : moderate resistance;

- F12 $\geq 125 \mathrm{MPa}$ : high resistance.

Longitudinal modulus of elasticity (in $\mathrm{MPa}$ )

Longitudinal modulus of elasticity $\left(\mathrm{E}_{\mathrm{L}}\right.$ is determined on woods at $12 \%$ moisture content and is a very important property for structural end-uses where pieces of wood usually support static bending forces in their largest direction, parallel to the fibres. This property characterises the relationship between load and deflection. It is an indicator of wood stiffness.

Longitudinal modulus of elasticity classification:

- $\mathrm{EL} \leq 12,500 \mathrm{MPa}$ : low modulus;

-12,500 MPa $\leq \mathrm{EL} \leq 18,500 \mathrm{MPa}$ : medium modulus;

- $\mathrm{EL} \geq 18,500 \mathrm{MPa}$ : high modulus.

\section{Natural durability and treatability}

Except special note concerning sapwood, durability characteristics refer to the heartwood of mature woods. Sapwood is always considered as non-durable towards biological wood decaying agents. A wood whose in-service moisture content is less than around $20 \%$ is not prone to fungal attack. Temperatures below around $5{ }^{\circ} \mathrm{C}$ prevent any fungal propagation. The same applies for woods under water or placed at high temperatures (around $60^{\circ} \mathrm{C}$ ), that are never attacked by decay, whatever their natural durability.

\section{Resistance to decay}

Resistance towards decay is determined on standardized samples in the presence of fungal strains, under controlled ambient conditions. These tests last several months.

The NF EN 350 standard, under review when this atlas was published, defines the classes of natural durability towards wood-decaying fungi:

- Highly durable woods: DC1 (durability class 1, called "class 1";

- Durable woods: DC2, called "class 2";

- Moderately durable woods: DC3, called "class 3";

- Poorly durable woods: DC4, called "class 4";

- Non-durable woods: DC5, called "class 5";

Resistance to dry wood insects (Lyctus, furniture beetle, death watch beetle)

Most commercialised tropical woods are not attacked by dry wood insects, provided that they do not contain sapwood. When the sapwood is not very demarcated, it is advisable to treat the wood against dry wood insects. Some tropical species are completely attacked in every part of the wood and require special attention when dry. Sawn woods or end-products are attacked only when they contain some sapwood and sufficient starch content.

Based on the NF EN 350 standard, a species is classified as sensitive (DC class S, called "class $\mathrm{S}^{\prime \prime}$ ) if it is attacked during laboratory tests. Otherwise, it is considered as durable (DC class D, called "class D").

\section{Resistance to termites}

Resistance to termites is determined in the same manner as for decay. Standardised samples are placed with termites. The intensity of termite attack, and consequently the natural resistance of 
the woods, is quantified by assessing the depth of the termites' penetration into the wood. The NF EN 350 standard defines three classes of natural durability against termites:

- Durable woods: DC class D (durability class D), called "class D";

- Moderately durable woods: DC class M, called "class M";

- Sensitive woods: DC class S, called "class S".

Heartwood treatability

Treatability corresponds to a wood's ability to be impregnated by a preservative product. The NF EN 350 standard defines four treatability classes:

- Class 1 - treatable. Sawn wood can be completely and easily impregnated with a treatment under pressure;

- Class 2 - moderately treatable. Complete penetration is usually not possible. However after a treatment under pressure during two or three hours, lateral penetration of more than $6 \mathrm{~mm}$ can be achieved for softwoods. For hardwoods, a high proportion of the structure (vessels and rays) can be impregnated;

- Class 3 - poorly treatable. Treatment under pressure for three or four hours cannot achieve a lateral penetration of more than 3-6 mm;

- Class 4 - non-treatable. Very little preservative product is absorbed, even after a treatment under pressure of 3-4 hours. Very little lateral and longitudinal penetration.

\section{Uses class}

The uses class corresponds to the degree of exposure to biological decay agents resulting from the service situation of a wooden item or structure. This class may change according to the design or the situation of the structure. It does not systematically define the service life, only the conditions of a potential biological attack. In a use class, the treatment specifications and the choice of the species have a direct effect on service life.

Thus, the service life must be interpreted according to the species and exposure severity. it depends on the wood's natural durability, but also on numerous others factors: design details of the structure (risks of water traps, ventilation of wood, etc.), type of maintenance scheduled, local climate conditions, etc.

The use of a wood whose natural durability is greater than the durability recommended by the NF EN 460 standard (July 1994) for a given use allows the structure's service life to be extended. Conversely, for structural elements with a very short service life (temporary construction), species with a lower natural durability than the durability mentioned in the EN 460 standard can be used.

Note It is important to avoid confusion between the "resistance to decay class" and "uses class", as the classification tables are different.

Service situations are grouped into use classes (see the NF EN 335 standard of May 2013). Each class corresponds to a category of uses associated with the same level of biological decay risks.

\section{Use class categories}

\begin{tabular}{|l|l|}
\hline Use class & General use \\
\hline $\mathbf{1}$ & Indoors in dry conditions \\
\hline $\mathbf{2}$ & $\begin{array}{l}\text { Indoors or under shelter, not exposed to adverse weather. Water condensation possible } \\
\text { Outdoors, not in contact with the ground, exposed to adverse weather. Class } 3 \text { can be subdivided } \\
\text { into two classes: } 3.1 \text { Brief exposure to dampening - 3.2 Prolonged exposure to dampening }\end{array}$ \\
\hline $\mathbf{4}$ & Outdoors, in contact with the ground or freshwater \\
\hline $\mathbf{5}$ & Regularly or permanently submerged in salt water \\
\hline
\end{tabular}

Specificities of Class 5. For concerned species, class 5 membership is indicated separately. A given species covering class 5 usually also covers class 4 , with the exception of a few species 
that only cover class 3 or class 2 (Basralocus, Garapa, Iroko, Louro vermelho and Sougué). The European NF EN 460 standard (July 1994) proposes a look-up table between the natural durability solid woods and their possible uses in a given risk class (see table below). This standard predates the replacement of "risk class" by "use class" (NF EN 335 of May 2013). These two concepts are nearly identical.

\section{Natural durability class according to risk class}

\begin{tabular}{|c|c|c|c|c|c|}
\hline \multirow{2}{*}{$\begin{array}{l}\text { Risk class covered by } \\
\text { natural durability }\end{array}$} & \multicolumn{5}{|c|}{ Natural durability class } \\
\hline & 1 & 2 & 3 & 4 & 5 \\
\hline 1 & $Y^{(1)}$ & Yes & Yes & Yes & Yes \\
\hline 2 & Yes & Yes & Yes & Yes but & Yes but \\
\hline 3 & Yes & Yes & Yes but & Case by case $^{(3)}$ & Case by case \\
\hline 4 & Yes & Yes but ${ }^{(2)}$ & No but ${ }^{(4)}$ & $\mathrm{No}^{(5)}$ & No \\
\hline 5 & Yes & No but & No but & No & No \\
\hline
\end{tabular}

(1) Yes: natural durability covers the risk class.

(2) Yes but: natural durability normally covers the risk class, but for certain uses, preservation treatment may be recommended.

(3) Case by case: natural durability may be sufficient, but depending on the species of wood, its permeability and end use, preservation treatment may be required.

(4) No but: preservation treatment is normally recommended, but for certain uses, the natural durability may be sufficient to cover the risk class.

(5) No: natural durability does not cover the risk class; preservation treatment is necessary.

For class risk 2 to 5, these correspondences are not clearly defined for certain durability levels. For certain species, use classes are given only as an indication. Corresponding values must be used with caution and professionalism.

\section{Preservation treatment}

This section recommends treatments or precautions for use of species where there is a risk of dry wood borer attacks and/or temporary or permanent humidification.

\section{Drying}

The general behaviour during kiln drying is described and assessed qualitatively. For certain species, safe practices for ensuring good drying quality are stated.

\section{Risk of deformation}

Sawn wood can be subject to 4 types of deformation (figure 2).
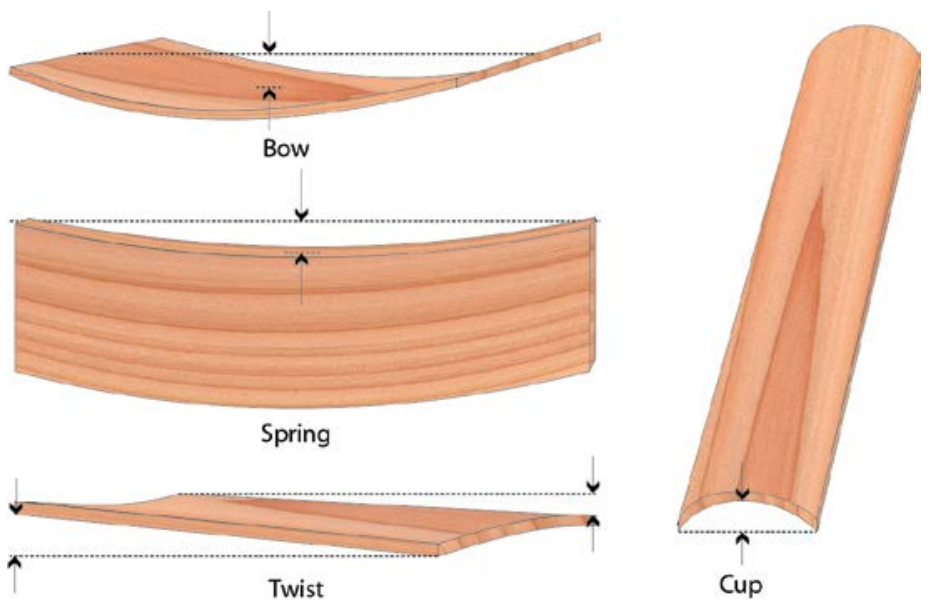

Figure 2. Types of wood deformation. 


\section{Risk of casehardening}

Casehardening corresponds to a blockage of water transfer from the inside to the outside of a piece of wood due to a change in peripheral wood cell structure. It is often caused by excessively rapid drying which results in overdrying the surface. It may be accompanied by the formation of "water pockets" within the wood.

\section{Risk of checking}

Checks can appear on the surface or ends of a piece of wood. They can also form inside sawn wood (internal checks).

\section{Risk of collapse}

Collapse is associated with the distortion of wood cells during the first phase of drying. This defect develops before the wood has reached the fibre saturation point (FSP). It appears in the form of rippling on the sawn side of the wood or complete deformation of the wood's surface, with or without internal checking.

\section{Drying schedules}

A drying schedule defines the series of climate conditions that must be followed in the kiln. In a wood drying schedule, the transition from one climate to the next is determined by the drop in the wood's moisture content.

For each species, one of the nine drying schedules presented at the end of this section is provided as an indication for use in a conditioned hot air dryer.

Each schedule is composed of five successive phases: two pre-heating phases, drying, conditioning and cooling.

The programmes are defined by five parameters:

- The duration of each phase (in hours);

- The moisture content of the wood $(\mathrm{M} \%)$, most often measured using electric probes, similar in principle to a pin moisture meter, inserted in several pieces of wood which are distributed in the kiln. The drying process can be controlled using an average of the values recorded by the probes, the highest recorded value, or the lowest value. Probes indicating outliers can be excluded from the calculation;

- The dry temperature, $\mathrm{T}\left({ }^{\circ} \mathrm{C}\right)$ required in the kiln. This must be regulated with sufficient accuracy to limit variations in temperature around the set point;

- Relative humidity, RH (\%);

- The equilibrium moisture content (EMC) (or UGL in German - "U" for wood moisture, GL for Gleichgewicht) corresponds to the moisture content which a wood tends to have in stable climatic conditions (temperature and relative air moisture).

A wood drying kiln is controlled by determining either the wood's equilibrium moisture content or relative air moisture. Equilibrium moisture content can be deduced from RH and T by using the Hailwood-Horrobin equation $(E M C=f(R H)(T)$. To do so, a calculation program is used, or a chart directly, based on these calculations.

The nine drying schedules provided here were designed with Gérard Gandon (Olergie), using schedules published by Cathild Industrie as reference.

For each species, the drying schedule is given as an indication for woods less than $35 \mathrm{~mm}$ thick. These guidelines must be validated through proper implementation. For woods between 35-55 mm thick, relative air moisture should be increased by $5 \%$ for each drying phase: for example, $30 \%$ for wood that is $27 \mathrm{~mm}$ thick and $35 \%$ for wood that is $41 \mathrm{~mm}$ thick. The temperature should be lowered by $3{ }^{\circ} \mathrm{C}$ for each phase of pre-heating, drying and conditioning.

For wood that is more than $55 \mathrm{~mm}$ thick, relative air moisture should be increased by $10 \%$ for each drying phase, and the temperature lowered by $5^{\circ} \mathrm{C}$ for each phase of pre-heating, drying and conditioning. 


\section{Drying schedule 1}

\begin{tabular}{|c|c|c|c|c|c|}
\hline Phases & Duration (h) & $\begin{array}{l}\text { Moisture content } \\
(\%) \text { probes }\end{array}$ & $\mathrm{T}\left({ }^{\circ} \mathrm{C}\right)$ & RH (\%) & UGL (\%) \\
\hline Pre-heating 1 & & $>50$ & 60 & 81 & 14.0 \\
\hline Pre-heating 2 & 3 & $>50$ & 65 & 76 & 12.0 \\
\hline \multirow[t]{12}{*}{ Drying } & & $>50$ & 68 & 68 & 10.0 \\
\hline & & $50-40$ & 70 & 63 & 9.1 \\
\hline & & $40-35$ & 70 & 61 & 8.7 \\
\hline & & $35-30$ & 70 & 56 & 7.9 \\
\hline & & $30-27$ & 72 & 50 & 7.0 \\
\hline & & $27-24$ & 72 & 44 & 6.3 \\
\hline & & $24-21$ & 75 & 39 & 5.5 \\
\hline & & $21-18$ & 75 & 34 & 4.9 \\
\hline & & $18-15$ & 75 & 29 & 4.3 \\
\hline & & $15-12$ & 80 & 28 & 3.9 \\
\hline & & $12-09$ & 80 & 24 & 3.4 \\
\hline & & $09-06$ & 80 & 22 & 3.2 \\
\hline Conditioning & 6 & & 73 & (3) & (2) \\
\hline Cooling & (1) & & Stop & (3) & (2) \\
\hline
\end{tabular}

\section{Drying schedule 2}

\begin{tabular}{|c|c|c|c|c|c|}
\hline Phases & Duration (h) & $\begin{array}{l}\text { Moisture con- } \\
\text { tent }(\%) \text { probes }\end{array}$ & $\mathrm{T}\left({ }^{\circ} \mathrm{C}\right)$ & RH (\%) & UGL (\%) \\
\hline Pre-heating 1 & & $>50$ & 58 & 84 & 15.0 \\
\hline Pre-heating 2 & 3 & $>50$ & 63 & 81 & 13.5 \\
\hline \multirow[t]{12}{*}{ Drying } & & $>50$ & 65 & 72 & 11.0 \\
\hline & & $50-40$ & 68 & 68 & 10.1 \\
\hline & & $40-35$ & 68 & 62 & 9.0 \\
\hline & & $35-30$ & 70 & 60 & 8.5 \\
\hline & & $30-27$ & 72 & 54 & 7.6 \\
\hline & & $27-24$ & 72 & 50 & 7.0 \\
\hline & & $24-21$ & 74 & 43 & 6.1 \\
\hline & & $21-18$ & 74 & 36 & 5.2 \\
\hline & & $18-15$ & 75 & 31 & 4.5 \\
\hline & & $15-12$ & 75 & 28 & 4.2 \\
\hline & & $12-09$ & 75 & 25 & 3.8 \\
\hline & & $09-06$ & 75 & 24 & 3.6 \\
\hline Conditioning & 6 & & 68 & (3) & (2) \\
\hline Cooling & (1) & & Stop & (3) & (2) \\
\hline
\end{tabular}

(1) Cooling: until the temperature inside the kiln no longer exceeds external temperature by more than $30^{\circ} \mathrm{C}$.

(2) $U G L=$ final $H \% \times 0.8$ to 0.9 .

(3) Subtract RH from the UGL determined in (2) and temperature, using the Hailwood-Horrobin equation. 


\section{Drying schedule 3}

\begin{tabular}{|c|c|c|c|c|c|}
\hline Phases & Duration (h) & $\begin{array}{l}\text { Moisture con- } \\
\text { tent }(\%) \text { probes }\end{array}$ & $\mathbf{T}\left({ }^{\circ} \mathbf{C}\right)$ & RH (\%) & UGL (\%) \\
\hline Pre-heating 1 & & $>50$ & 55 & 84 & 15.5 \\
\hline Pre-heating 2 & 3 & $>50$ & 57 & 83 & 15.0 \\
\hline \multirow[t]{12}{*}{ Drying } & & $>50$ & 60 & 76 & 12.5 \\
\hline & & $50-40$ & 60 & 73 & 11.6 \\
\hline & & $40-35$ & 60 & 69 & 10.7 \\
\hline & & $35-30$ & 60 & 62 & 9.5 \\
\hline & & $30-27$ & 63 & 55 & 8.2 \\
\hline & & $27-24$ & 64 & 50 & 7.5 \\
\hline & & $24-21$ & 65 & 46 & 6.9 \\
\hline & & $21-18$ & 65 & 39 & 6.0 \\
\hline & & $18-15$ & 68 & 32 & 5.0 \\
\hline & & $15-12$ & 70 & 29 & 4.5 \\
\hline & & $12-09$ & 70 & 25 & 4.0 \\
\hline & & $09-06$ & 70 & 24 & 3.9 \\
\hline Conditioning & 6 & & 63 & (3) & (2) \\
\hline Cooling & (1) & & Stop & (3) & (2) \\
\hline
\end{tabular}

(1) Cooling: until the temperature inside the kiln no longer exceeds external temperature by more than $30{ }^{\circ} \mathrm{C}$.

(2) $U G L=$ final $H \% \times 0.8$ to 0.9 .

(3) Subtract RH from the UGL determined in (2) and temperature, using the Hailwood-Horrobin equation.

\section{Drying schedule 4}

\begin{tabular}{|c|c|c|c|c|c|}
\hline Phases & Duration (h) & $\begin{array}{l}\text { Moisture con- } \\
\text { tent }(\%) \text { probes }\end{array}$ & $\mathrm{T}\left({ }^{\circ} \mathrm{C}\right)$ & RH (\%) & UGL (\%) \\
\hline Pre-heating 1 & & $>50$ & 50 & 86 & 16.5 \\
\hline Pre-heating 2 & 3 & $>50$ & 52 & 85 & 16.0 \\
\hline \multirow[t]{12}{*}{ Drying } & & $>50$ & 55 & 82 & 14.7 \\
\hline & & $50-40$ & 55 & 80 & 13.8 \\
\hline & & $40-35$ & 55 & 75 & 12.6 \\
\hline & & $35-30$ & 56 & 73 & 12.0 \\
\hline & & $30-27$ & 58 & 67 & 10.5 \\
\hline & & $27-24$ & 60 & 58 & 8.9 \\
\hline & & $24-21$ & 62 & 50 & 7.5 \\
\hline & & $21-18$ & 64 & 45 & 6.8 \\
\hline & & $18-15$ & 65 & 37 & 5.7 \\
\hline & & $15-12$ & 65 & 34 & 5.3 \\
\hline & & $12-09$ & 65 & 28 & 4.5 \\
\hline & & $09-06$ & 65 & 24 & 4.0 \\
\hline Conditioning & 6 & & 58 & (3) & (2) \\
\hline Cooling & (1) & & Stop & (3) & (2) \\
\hline
\end{tabular}

(1) Cooling: until the temperature inside the kiln no longer exceeds external temperature by more than $30{ }^{\circ} \mathrm{C}$.

(2) $U G L=$ final $H \% \times 0.8$ to 0.9 .

(3) Subtract RH from the UGL determined in (2) and temperature, using the Hailwood-Horrobin equation. 


\section{Drying schedule 5}

\begin{tabular}{|c|c|c|c|c|c|}
\hline Phases & Duration $(\mathbf{H})$ & $\begin{array}{l}\text { Moisture con- } \\
\text { tent }(\%) \text { probes }\end{array}$ & $\mathrm{T}\left({ }^{\circ} \mathrm{C}\right)$ & RH (\%) & UGL (\%) \\
\hline Pre-heating 1 & & $>50$ & 50 & 87 & 17.0 \\
\hline Pre-heating 2 & 4 & $>50$ & 50 & 86 & 16.5 \\
\hline \multirow[t]{12}{*}{ Drying } & & $>50$ & 53 & 83 & 15.2 \\
\hline & & $50-40$ & 53 & 80 & 14.1 \\
\hline & & $40-35$ & 54 & 80 & 13.9 \\
\hline & & $35-30$ & 55 & 75 & 12.5 \\
\hline & & $30-27$ & 57 & 70 & 11.0 \\
\hline & & $27-24$ & 58 & 61 & 9.4 \\
\hline & & $24-21$ & 59 & 51 & 7.9 \\
\hline & & $21-18$ & 60 & 47 & 7.3 \\
\hline & & $18-15$ & 61 & 39 & 6.1 \\
\hline & & $15-12$ & 62 & 35 & 5.6 \\
\hline & & $12-09$ & 62 & 30 & 5.0 \\
\hline & & $09-06$ & 62 & 26 & 4.4 \\
\hline Conditioning & 8 & & 55 & (3) & (2) \\
\hline Cooling & (1) & & Stop & (3) & (2) \\
\hline
\end{tabular}

(1) Cooling: until the temperature inside the kiln no longer exceeds external temperature by more than $30^{\circ} \mathrm{C}$.

(2) $U G L=$ final $H \% \times 0.8$ to 0.9 .

(3) Subtract RH from the UGL determined in (2) and temperature, using the Hailwood-Horrobin equation.

\section{Drying schedule 6}

\begin{tabular}{|c|c|c|c|c|c|}
\hline Phases & Duration $(\mathbf{H})$ & $\begin{array}{l}\text { Moisture con- } \\
\text { tent }(\%) \text { probes }\end{array}$ & $\mathbf{T}\left({ }^{\circ} \mathrm{C}\right)$ & RH (\%) & UGL (\%) \\
\hline Pre-heating 1 & & $>50$ & 50 & 87 & 17.0 \\
\hline Pre-heating 2 & 4 & $>50$ & 50 & 86 & 16.5 \\
\hline \multirow[t]{12}{*}{ Drying } & & $>50$ & 53 & 85 & 15.7 \\
\hline & & $50-40$ & 53 & 82 & 14.6 \\
\hline & & $40-35$ & 54 & 78 & 13.4 \\
\hline & & $35-30$ & 55 & 77 & 12.9 \\
\hline & & $30-27$ & 57 & 73 & 11.9 \\
\hline & & $27-24$ & 58 & 68 & 10.7 \\
\hline & & $24-21$ & 60 & 61 & 9.3 \\
\hline & & $21-18$ & 62 & 52 & 7.9 \\
\hline & & $18-15$ & 64 & 43 & 6.6 \\
\hline & & $15-12$ & 65 & 39 & 6.0 \\
\hline & & $12-09$ & 65 & 31 & 5.0 \\
\hline & & $09-06$ & 65 & 28 & 4.5 \\
\hline Conditioning & 8 & & 58 & (3) & (2) \\
\hline Cooling & (1) & & Stop & (3) & (2) \\
\hline
\end{tabular}

(1) Cooling: until the temperature inside the kiln no longer exceeds external temperature by more than $30^{\circ} \mathrm{C}$.

(2) $U G L=$ final $H \% \times 0.8$ to 0.9 .

(3) Subtract RH from the UGL determined in (2) and temperature, using the Hailwood-Horrobin equation. 


\section{Drying schedule 7}

\begin{tabular}{|c|c|c|c|c|c|}
\hline Phases & Duration $(\mathbf{H})$ & $\begin{array}{l}\text { Moisture con- } \\
\text { tent }(\%) \text { probes }\end{array}$ & $\mathbf{T}\left({ }^{\circ} \mathbf{C}\right)$ & RH (\%) & UGL (\%) \\
\hline Pre-heating 1 & & $>50$ & 40 & 86 & 17.0 \\
\hline Pre-heating 2 & 4 & $>50$ & 43 & 85 & 16.5 \\
\hline \multirow[t]{12}{*}{ Drying } & & $>50$ & 45 & 83 & 15.7 \\
\hline & & $50-40$ & 45 & 80 & 14.6 \\
\hline & & $40-35$ & 45 & 77 & 13.8 \\
\hline & & $35-30$ & 45 & 74 & 12.9 \\
\hline & & $30-27$ & 47 & 69 & 11.5 \\
\hline & & $27-24$ & 49 & 61 & 9.9 \\
\hline & & $24-21$ & 50 & 52 & 8.4 \\
\hline & & $21-18$ & 53 & 48 & 7.7 \\
\hline & & $18-15$ & 56 & 41 & 6.6 \\
\hline & & $15-12$ & 59 & 36 & 5.9 \\
\hline & & $12-09$ & 61 & 30 & 5.0 \\
\hline & & $09-06$ & 65 & 29 & 4.7 \\
\hline Conditioning & 8 & & 58 & (3) & (2) \\
\hline Cooling & (1) & & Stop & (3) & (2) \\
\hline
\end{tabular}

(1) Cooling: until the temperature inside the kiln no longer exceeds external temperature by more than $30{ }^{\circ} \mathrm{C}$.

(2) $U G L=$ final $H \% \times 0.8$ to 0.9 .

(3) Subtract RH from the UGL determined in (2) and temperature, using the Hailwood-Horrobin equation.

\section{Drying schedule 8}

\begin{tabular}{|c|c|c|c|c|c|}
\hline Phases & Duration (H) & $\begin{array}{l}\text { Moisture con- } \\
\text { tent }(\%) \text { probes }\end{array}$ & $\mathrm{T}\left({ }^{\circ} \mathrm{C}\right)$ & RH (\%) & UGL (\%) \\
\hline Pre-heating 1 & & $>50$ & 45 & 86 & 17.0 \\
\hline Pre-heating 2 & 4 & $>50$ & 45 & 85 & 16.5 \\
\hline \multirow[t]{12}{*}{ Drying } & & $>50$ & 48 & 84 & 15.7 \\
\hline & & $50-40$ & 48 & 80.5 & 14.6 \\
\hline & & $40-35$ & 49 & 77 & 13.4 \\
\hline & & $35-30$ & 50 & 75 & 12.9 \\
\hline & & $30-27$ & 51 & 70 & 11.5 \\
\hline & & $27-24$ & 53 & 62 & 9.9 \\
\hline & & $24-21$ & 54 & 53 & 8.4 \\
\hline & & $21-18$ & 55 & 48.5 & 7.7 \\
\hline & & $18-15$ & 55 & 40 & 6.6 \\
\hline & & $15-12$ & 55 & 35 & 5.9 \\
\hline & & $12-09$ & 60 & 30 & 5.0 \\
\hline & & $09-06$ & 60 & 28 & 4.7 \\
\hline Conditioning & 8 & & 58 & (3) & (2) \\
\hline Cooling & (1) & & Stop & (3) & (2) \\
\hline
\end{tabular}

(1) Cooling: until the temperature inside the kiln no longer exceeds external temperature by more than $30^{\circ} \mathrm{C}$.

(2) $U G L=$ final $H \% \times 0.8$ to 0.9 .

(3) Subtract RH from the UGL determined in (2) and temperature, using the Hailwood-Horrobin equation. 


\section{Drying schedule 9}

\begin{tabular}{|c|c|c|c|c|c|}
\hline Phases & Duration $(\mathrm{H})$ & $\begin{array}{l}\text { Moisture con- } \\
\text { tent }(\%) \text { probes }\end{array}$ & $\mathbf{T}\left({ }^{\circ} \mathrm{C}\right)$ & RH (\%) & UGL (\%) \\
\hline Pre-heating 1 & & $>40$ & 35 & 87 & 18.0 \\
\hline Pre-heating 2 & 6 & $>40$ & 38 & 85 & 17.0 \\
\hline \multirow[t]{10}{*}{ Drying } & & $>40$ & 41 & 82 & 15.7 \\
\hline & & $40-35$ & 44 & 81 & 15.0 \\
\hline & & $35-30$ & 46 & 80 & 14.5 \\
\hline & & $30-27$ & 48 & 77 & 13.5 \\
\hline & & $27-24$ & 50 & 72 & 12.0 \\
\hline & & $24-21$ & 52 & 63 & 10.0 \\
\hline & & $21-18$ & 54 & 54 & 8.5 \\
\hline & & $18-15$ & 56 & 47 & 7.4 \\
\hline & & $15-12$ & 58 & 41 & 6.5 \\
\hline & & $12-09$ & 60 & 34 & 5.6 \\
\hline Conditioning & 8 & & 55 & (3) & (2) \\
\hline Cooling & (1) & & Stop & (3) & (2) \\
\hline
\end{tabular}

(1) Cooling: until the temperature inside the kiln no longer exceeds external temperature by more than $30^{\circ} \mathrm{C}$.

(2) $U G L=$ final $H \% \times 0.8$ to 0.9 .

(3) Subtract RH from the UGL determined in (2) and temperature, using the Hailwood-Horrobin equation.

\section{Sawing and machining}

\section{Blunting effect}

This effect is essentially related to the silica content of wood and its hardness; it determines the type of tools to be used for sawing and machining.

\section{Sawteeth}

Depending on a wood's blunting effect, ordinary or alloy steel, or stellite-tipped sawteeth should be used.

\section{Machining tools}

The choice of tools is defined according to the wood's abrasiveness, i.e. its blunting effect. If there is no problem, normal tools can be used. Otherwise, special tools with high-speed steel (HSS) or tungsten carbide are recommended.

Some species contain chemical substances which can represent varying degrees of toxicity for users during processing operations (sawdust during sawing or machining) and which may cause skin or mucous allergies and in some extreme cases, respiratory disorders. These disorders can be significantly limited and even avoided by using protective equipment (gloves, masks), and by fitting efficient aspiration systems (legal requirements).

\section{Suitability for peeling}

Usually, species used for peeling are soft to moderately hard. Logs are well-formed and without defects to achieve a good output. Moreover, the wood must display good behaviour during drying in order to limit the risks of splitting and veneer deformation.

\section{Suitability for slicing}

Woods used for slicing must present specific aesthetic qualities such as: colour, figuring, fine texture and a ribbon-like appearance.

\section{Assembling}

\section{Nailing and screwing}

It is important to know whether it is easy or not to drive a nail or screw without the risk of splitting. Otherwise, pre-boring is recommended.

Moreover, the wood's ability to hold a nail or a screw is mentioned: good or poor. 


\section{Gluing}

In the wood industry, gluing - of tropical woods in particular - has made the most progress since the 1980s. The appearance on the market of new and increasingly effective adhesives makes it possible to glue any type of wood unconditionally, whatever the characteristics, and meet growing requirements in terms of water resistance and mechanical strength.

These technological advances improve how tropical woods are used by making it possible to glue together cuts obtained from secondary species, crooked or small-diameter logs, wood with significant defects, downgraded wood and sawmill waste.

Products like glue laminated timber are stable and homogenous. The use of gluing techniques to combine very different species offers new prospects for increasing the use of wood in high end projects. Gluing wood to other materials is possible.

However gluing can be made difficult by the characteristics of certain species and the need to respect the code of practice. This applies to the high-density species described in this atlas (wood with an average density of over 0.80).

Studies carried out by CIRAD have shown that gluing wood with significant defects or seemingly unfavourable characteristics can yield satisfactory results. However, recommendations must be followed. The adhesive must be chosen in relation to the end use of the product, the production system, the time needed for assembling and the desired pressing time.

\section{Commercial grading}

\section{Sawn timber appearance grading (temperate woods) \\ Principle}

The criteria generally used to grade wood are the presence and extent of defects (knots, splits, resin pockets, grain gradient, wanes, biological deterioration, geometric deformations, etc.). All of these defects are likely to affect the aesthetic properties of the woods and to reduce the volume of wood that can actually be used. Some of these defects may be authorised, but will cause a reduction the area or volume of wood used as the basis for calculating the price (boules), or may require a change of category (downgrading) without modifying the area or volume (square-edged timber).

\section{Rules}

The aspect grading, described in the NF EN 975-1 standard (April 2009) concerns sawn European Oak and European Beech. The NF EN 975-2 standard (November 2004) concerns sawn poplar.

The NF EN 1611-1 standard (October 1999) concerns sawn Spruce, Fir, Pine and Douglas Fir. The NF EN 1611-1/A1 standard (March 2003) adds European Larch to this list, without altering the grading criteria.

\section{Special case of Western Red Cedar}

Published by the Pacific Lumber Inspection Bureau (PLIB, 2003), the Export R-List Grading and Dressing Rules presents American grading rules regarding the following species: Douglas Fir, Pacific coast (West coast) Hemlock and true Firs, Sitka Spruce and Red Cedar.

The Canadian grading rules of the NLGA (National Lumber Grades Authority) concern Canadian timber, and in particular Red Cedar (January 2008).

\section{Sawn timber appearance grading (tropical woods)}

\section{Principle}

For tropical wood, there are two methods of timber appearance grading:

- grading of the considered part according to the number of "standard" defects that it presents, relative to its dimensions (the larger the part's area, the greater the number of defects tolerated). This is the current grading principle for African square-edged lumber (ATIBT riles, 1999 edition), progressively superseded by SATA (Sciages Avivés Tropicaux Africains) rules. 
- clean cut grading. The percentage of rectangular defect-free areas is calculated relative to the area of the part. We thus obtain a percentage of defects, from which the part choice is defined.

For SATA rules, a first choice (i) must present a 90\% defect-free area. A second choice (ii) must present a $80 \%$ defect-free area and so on and so forth, with a variable number of cuts depending on the area of the part.

This principle is used by the Malaysia Grading Rules (MGR) and the National Hardwood Lumber Association rules (NHLA).

\section{Rules}

The SATA grading rules were defined in 1976, under the aegis of the Commission of European Communities, at the instance of the following 5 African states: Cameroon, Congo, Côte d'Ivoire, Gabon and the Democratic Republic of the Congo. The study was entrusted to the Centre Technique Forestier Tropical in Nogent-sur-Marne (CTFT - French: Centre for Tropical Forests, that was merged, along with other institutes, with the CIRAD in 1984). These rules were republished in 1996 in "SATA ("Sciages Avivés Tropicaux Africains"): grading rules".

The NHLA grading rules were originally drawn up for North American hardwood species. The current version (2015) also covers tropical species: "Rules for the Measurement \& Inspection of Hardwood \& Cypress". It is available from the NHLA website.

The "Bois guyanais classés" rules (graded Guyanese woods) (1990) were defined for wood used and processed in French Guiana. They are mainly used on the Guyanese market or exported to the French West Indies.

The MGR rules were drawn up in 1968, then revised in 1984 and in July 2009. They pertain to Malaysian species, but may be used for wood from other parts of Asia.

\section{Visual structure grading}

In this atlas, visual structure grading of tropical woods and hardwoods is based both on French standards (NF B 52-001-1 (August 2011), NF B 52-001-1/A1 (April 2013) and NF B 52-001/ A2 (February 2015)) and the European EN 1912 (2012) standard, together with corresponding national standards. In this atlas, 105 of the species listed are graded for mechanical strength using visual grading methods according to rules from one or several of these standards.

\section{Fire safety}

Two distinct wood properties characterise its fire behaviour: fire resistance and reaction to fire.

Fire resistance is the time during which a material exposed to fire will continue to provide its initial functions within the structure: mechanical stability for the structure, fire stop or fire break properties of a door, etc.

Reaction to fire characterizes the production of flames, heat, fumes, particles and drops of material exposed to fire. Reaction to fire concerns mainly those materials used for floors, walls and ceilings, that are in contact with individuals.

Information provided in this atlas pertains to reaction to fire.

\section{Conventional French grading}

French regulations (conventional grading - Order of 21 November 2002) define six classes of reaction to fire:

- M0 - non-combustible;

- M1 - non-flammable;

- M2 - poorly flammable;

- M3 - moderately flammable;

- M4 - readily flammable;

- M5 - highly flammable; 
In general terms, the grading of untreated solid woods is as follows:

Solid hardwoods:

- thicknesses greater than or equal to $14 \mathrm{~mm}$ : M3 - moderately flammable;

- thicknesses less than 14 mm: M4 - readily flammable;

Solid softwoods:

- thicknesses greater than or equal to $18 \mathrm{~mm}$ : M3 - moderately flammable;

- thicknesses less than $18 \mathrm{~mm}$ : M3 - readily flammable;

\section{Euroclass grading}

European standardization (Euroclasses of the NF EN 13501-1 + A1 standard, February 2013) introduced a new classification based on different test methods.

- A1, A2, B, C, D, E and F for wall and ceiling coverings;

- $\mathrm{A} 1 \mathrm{fl}, \mathrm{A} 2 \mathrm{fl}, \mathrm{Bfl}, \mathrm{Cfl}, \mathrm{Dfl}, \mathrm{Efl}$ and $\mathrm{Ffl}$ for floor coverings.

The information provided in this atlas pertains to wall and ceiling coverings.

Within each class, two additional reaction to fire criteria characterize fume product on the one hand (s0, s1, s2) and the production of incandescent drops and particles on the other hand $(\mathrm{d} 0, \mathrm{~d} 1, \mathrm{~d} 2)$.

Considering the properties of the wood species most commonly used in Europe (NF EN 14081-1, April 2016), solid wood is, by convention, classed Ds2 d0 for wall and ceiling coverings if its density is greater than 0.35. If a wood's density is less than 0.35, the species is considered ungraded. This applies to the following species: Balsa, Emien, Essessang, Fromager, Pashaco, Sumauma.

Any more favourable grading must be justified by testing the species in this atlas. This applies to Larch and to the following five tropical species found in Latin America and Guyana: Mandioqueira, Pau roxo, Basralocus, Ipê and Louro vermelho.

\section{End uses}

A wood's potential uses are directly dependent upon its technological properties. The end uses mentioned in the list are not exhaustive. They cover the most commonly known end uses which must be validated through proper implementation. Certain uses are given as an indication only (traditional, regional or historical uses).

\section{Common names}

The principal local/common names used in the producing countries are mentioned, along with the trade names used in the importing countries when they are different from the ATIBT pilot names.

For each species described, the list of 'Common names' is not exhaustive. The result of very thorough compilation, a nearly exhaustive inventory of these common names is available on the website of the Forest Products Laboratory in Madison (United States). www.fpl.fs.fed.us/ search/commonname_request.php. 